\title{
Leader-Follower Based Locally Rigid Formation Control
}

\author{
Krishna Raghuwaiya, Bibhya Sharma $\mathbb{D}$, and Jito Vanualailai $\mathbb{D}$ \\ School of Computing, Information \& Mathematical Sciences, The University of the South Pacific, Suva, Fiji \\ Correspondence should be addressed to Bibhya Sharma; bibhya.sharma@usp.ac.fj
}

Received 4 August 2017; Accepted 5 December 2017; Published 25 February 2018

Academic Editor: Fernando García

Copyright (c) 2018 Krishna Raghuwaiya et al. This is an open access article distributed under the Creative Commons Attribution License, which permits unrestricted use, distribution, and reproduction in any medium, provided the original work is properly cited.

\begin{abstract}
This paper addresses motion control of a cooperative intelligent transport system (C-ITS) of nonholonomic mobile robots navigating a dynamic environment while maintaining a locally rigid formation. We consider the design of acceleration-based control inputs that govern the motion of cooperative intelligent transport system (C-ITS) using the artificial potential fields method for the avoidance of obstacles and attraction to designated targets. The control scheme utilizes a new leader-follower strategy using Cartesian coordinates to accomplish a collision-free locally rigid formation of an autonomous and intelligent transportation system. The concepts of virtual parking bays and minimum distance technique (MDT) are utilized to attain prescribed orientations of the formation at the final destination. The robustness of the control scheme is established by considering the effect noise on the formation, while the effectiveness of the proposed nonlinear control laws is demonstrated through computer simulations.
\end{abstract}

\section{Introduction}

Connected and autonomous vehicles play a huge role in the transportation industry worldwide and will govern the industry in the coming years. The connectedness is governed through vehicle-to-vehicle (V2V) and vehicle-toinfrastructure (V2I) communication, which is facilitated using wireless and cellular technologies at current times. Such key communications of the cooperative intelligent transport system (C-ITS) allow respective shareholders to utilize information and coordinate task transfers in a cooperative manner. The networked V2V and V2I interactions deliver substantial social benefits in terms of safer, traffic-efficient, cooperative autonomous driving and energy-efficient transportation systems on our roads and highways $[1,2]$.

Cooperative tasks are more efficiently performed with desired robustness using multiple robots, which may not be possible with single robots. The benefits become apparent when considering distributed tasks, dangerous or hazardous tasks, and tasks that contain redundancies and when providing flexibility to task execution and robustness of systems [38]. In addition, there is the added advantage that working with multiple robots is less expensive than with just one specialized robot. To date there have been various applications of the control of C-ITS, for example, collaborative mapping and planning, search and rescue operations, flocking and schooling, transportation of large objects, undersea and space exploration, target seeking, competitive games, service robots, and military reconnaissance and surveillance $[4,8-$ 13]. The ability of C-ITS to autonomously navigate in stable configurations while avoiding obstacles and collisions is also central to real-world applications $[8,14]$. In many applications, the execution of the task requires formation control [3], and the accomplishment of the overall operation depends on each mobile robot operating in a prescribed manner. Typical examples include carrying a heavy load on roads and highways, hunting, and enclosing on an enemy to name a few.

The concept of formation control of mechanical systems, an integrated branch of motion planning and control of CITS, is receiving unprecedented attention from researchers all over the world for real-world applications [14]. The basic idea of formation control algorithms is to ensure that a group of mobile robots move effectively as a whole to jointly perform certain task(s). Examples of formation control tasks include delegation of feasible formations, establishing formations, maintaining and mobilizing different formation shapes, and switching between formations [15]. There are numerous approaches in literature in relation to the observance of a prescribed formation of a flock during motion [14]; however, 
split/rejoin maneuvers and rigid formations are the prevalent approaches. The split/rejoin maneuvers are frequently cited in flocks of birds, swarms of insects and ants, and herds of animals. The applications of split/rejoin maneuvers in the field of robotics include reconnaissance, sampling, and surveillance. In contrast, rigid formations (globally rigid or locally rigid) are required in many engineering applications, for example, parallel and simultaneous transportation of vehicles or delivery of payloads $[12,14,16]$. Alterations or distortions in formation must be detected early by a system and allow for reconfiguration for continued operation [7] in a locally rigid formation.

In recent literature, a variety of control strategies have been proposed for formation or cooperative control of mobile robots and have been roughly categorized into virtual structure, behavior-based, and leader-follower schemes. The behavior-based approach normally [3] assigns different possible behaviors (e.g., formation keeping, obstacle avoidance, collision avoidance, and target attraction) to each individual robot. The ultimate action of each robot is determined by evaluating the comparative importance of each behavior. The limitation of the behavior-based approach is that it is difficult to analyze its behavioral performance mathematically and therefore it is difficult to guarantee system stability [17]. The virtual structures $[6,18-20]$ consider the entire formation as a single virtual rigid body. This networked structure can then be considered similar to a physical body and thus it is easy to maintain the formation of the whole group during maneuvers; that is, the virtual rigid body progresses as a whole in a given course with some given bearing. In the leader-follower approach [14, 17, 21-24], one robot in the multirobot formation, a virtual robot, is assigned the role of the leader to pursue some team objective, while the follower robots follow their designated leader. The follower robots then place themselves relative to the leader and maintain a predefined offset with a desired relative position [17]. In [25], Sharma et al. proposed a leader-follower scheme in a Lyapunov-based decentralized formation control planner for a swarm of 2-link mobile manipulators. The accelerationbased controllers ensured a locally rigid formation for the swarm. Locally rigid formation was achieved by integrating maximum and minimum interrobot distance bounds with desired headings in the control scheme.

In this paper, we adopt the architecture of the Lyapunovbased Control Scheme (LbCS) of [14], an artificial potential field (APF) method to design attractive and repulsive potential field functions to control the formation of C-ITS. An extension to [25], this paper presents a new set of nonlinear time-invariant control laws through an amalgamation of the LbCS and a new leader-follower scheme to maintain and mobilize a locally rigid formation. The limitation of the approach is twofold; firstly it comes with the disadvantages of APFs, in particular the local minima, and secondly the dependence on the lead robot and the poor disturbance rejection properties [26] of the leader-follower scheme. However, the novelty of the new approach lies in its ability to design continuous nonlinear control laws to translate locally rigid formations of nonholonomic systems tagged with dynamic constraints. The LbCS controllers are elegant

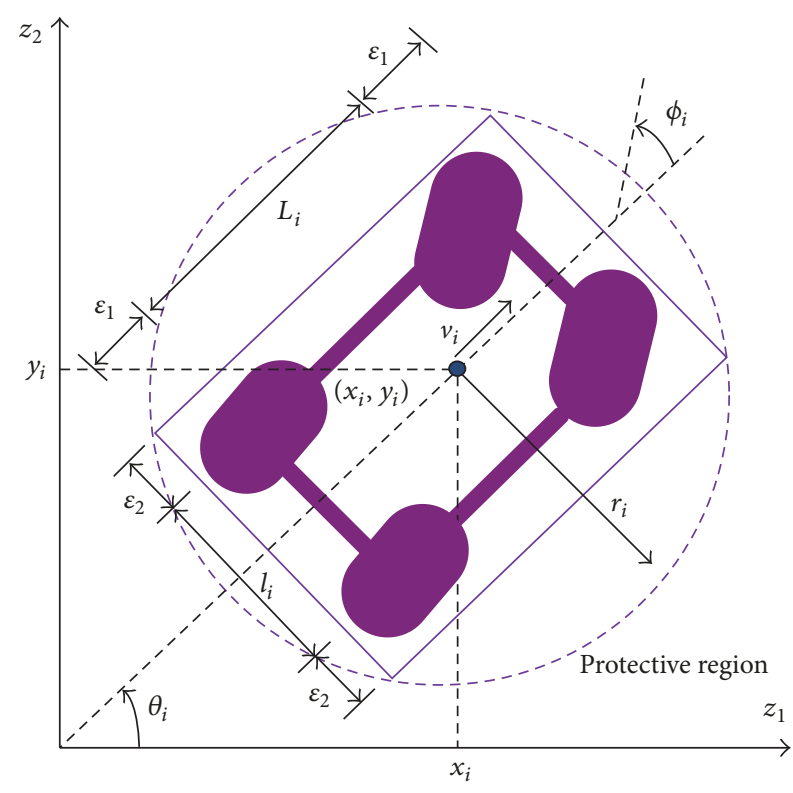

FIGURE 1: Kinematic model of the car-like mobile robot.

and very simple to construct compared to the mathematically and computationally intensive ones in literature, and LbCS has an in-built process of converting these constraints into artificial obstacles and incorporating them into the controllers. Finally, the virtual connectivity from the new leaderfollower scheme facilitates diverse tasks compared to merely cooperative agents [26].

The advantage of the proposed scheme is that it uses Cartesian coordinate representation to avoid any singular points as encountered when using polar coordinates, even though polar coordinate representation may still be simpler to use. Another advantage of the proposed scheme in this paper is that it has a dual frame methodology, a new leaderfollower approach, and the assignment of a single target for the group of vehicles. The overarching framework is a leader-follower scheme for C-ITS to establish, maintain, and translate the whole team in a locally rigid formation around the workplace performing a given task through centralized control laws. Finally, the treatment of several categories of obstacles is also included within the algorithm.

\section{Vehicle Model}

In this section, we derive a new kinematic model for the leader-follower based formation control of multiple vehicles. We will consider $n(n \in \mathbb{N})$ car-like vehicles in the Euclidean plane. We let $\mathscr{A}_{1}$ represent the leader and $\mathscr{A}_{i}$ for $i=2, \ldots, n$ take the role of follower robots. With reference to Figure 1, adopted from [27], and for $i \in\{1, \ldots, n\},\left(x_{i}, y_{i}\right)$ represents the Cartesian coordinates and gives the reference point of each vehicle and $\theta_{i}$ gives the orientation of the $i$ th car with respect to $z_{1}$-axis of the $z_{1} z_{2}$-plane. Moreover, $\phi_{i}$ gives the $i$ th vehicles steering angle with respect to its longitudinal axis. $L_{i}$ represents the distance between the centers of the front and rear axles of the $i$ th robot, and $l_{i}$ is the length of each axle. 
Next, to ensure that each vehicle safely steers past an obstacle, we adopt the nomenclature of [14] and construct circular regions that protect the robot. With reference to Figure 1, given the clearance parameters $\epsilon_{1}>0$ and $\epsilon_{2}>0$, we enclose each vehicle by a protective circular region centered at $\left(x_{i}, y_{i}\right)$ with radius $r_{i}=(1 / 2) \sqrt{\left(L_{i}+2 \epsilon_{1}\right)^{2}+\left(l_{i}+2 \epsilon_{2}\right)^{2}}$.

Furthermore, we assume no slippage condition of the rear and front wheels of the mobile robots of $\mathscr{A}_{i}$ when in contact with a rigid surface, that is, the lateral (or tangential) velocities of the wheels of the cars are assumed to be zero. We have no slippage (i.e., $\dot{x}_{i} \sin \theta_{i}-\dot{y}_{i} \cos \theta_{i}=0$ ) and pure rolling (i.e., $\dot{x}_{i} \cos \theta_{i}+\dot{y}_{i} \sin \theta_{i}=v_{i}$ ) of the car-like mobile robot which generate the nonholonomic constraints on the system. These assumptions of no slippage and pure rolling of the car-like mobile robots are essential to generate the nonholonomic constraints on the system. The kinematics of the system which inherently capture these nonholonomic constraints, adopted from [14], are

$$
\begin{aligned}
& \dot{x}_{i}=v_{i} \cos \theta_{i}-\frac{L_{i}}{2} \omega_{i} \sin \theta_{i}, \\
& \dot{y}_{i}=v_{i} \sin \theta_{i}+\frac{L_{i}}{2} \omega_{i} \cos \theta_{i}, \\
& \dot{\theta}_{i}=\frac{v_{i}}{L_{i}} \tan \phi_{i}:=\omega_{i}, \\
& \dot{v}_{i}:=\sigma_{i 1}, \\
& \dot{\omega}_{i}:=\sigma_{i 2},
\end{aligned}
$$

for $i \in\{1, \ldots, n\}$, and, without any loss of generality, we assume that $\phi_{i}=\theta_{i}$. In system (1), $v_{i}$ and $\omega_{i}$ are, respectively, the instantaneous translational and rotational velocities, while $\sigma_{i 1}$ and $\sigma_{i 2}$ are the instantaneous translational and rotational accelerations of the $i$ th robot.

Now, system (1) is a description of the instantaneous velocities and accelerations of $\mathscr{A}_{i}$. Let the vector $\mathbf{x}_{i}:=$ $\left(x_{i}, y_{i}, \theta_{i}, v_{i}, \omega_{i}\right) \in \mathbb{R}^{5}$ refer to the position $\left(x_{i}, y_{i}\right)$, orientation, $\theta_{i}$, and the velocities $\left(v_{i}, \omega_{i}\right)$ of $\mathscr{A}_{i}$ at time $t \geq 0$.

Now, let

$$
\begin{aligned}
\mathbf{f}_{i}\left(\mathbf{x}_{i}\right) & =\left(f_{i 1}\left(\mathbf{x}_{i}\right), f_{i 2}\left(\mathbf{x}_{i}\right), f_{i 3}\left(\mathbf{x}_{i}\right), 0,0\right) \\
& :=\left(\dot{x}_{i}, \dot{y}_{i}, \dot{\theta}_{i}, 0,0\right) \in \mathbb{R}^{5},
\end{aligned}
$$

and $\mathbf{u}_{i}(t):=\left(\sigma_{i 1}(t), \sigma_{i 2}(t)\right) \in \mathbb{R}^{2}$. Then system (1) can be written compactly as

$$
\dot{\mathbf{x}}_{i}:=\mathbf{f}_{i}\left(\mathbf{x}_{i}\right)+\mathbf{B}_{i} \mathbf{u}_{i}(t),
$$

where $\mathbf{B}_{i}$ is a $5 \times 2$ matrix of the form

$$
\mathbf{B}_{i}=\left[\begin{array}{ll}
0 & 0 \\
0 & 0 \\
0 & 0 \\
1 & 0 \\
0 & 1
\end{array}\right] .
$$

Let $\mathbf{x}:=\left(\mathbf{x}_{1}, \mathbf{x}_{2}, \ldots, \mathbf{x}_{n}\right) \in \mathbb{R}^{5 n}$ refer to the positions, orientations, and the velocities of all the vehicles in the C-ITS.

Let $\mathbf{f}(\mathbf{x}):=\left(\mathbf{f}_{1}(\mathbf{x}), \mathbf{f}_{2}(\mathbf{x}), \ldots, \mathbf{f}_{n}(\mathbf{x})\right) \in \mathbb{R}^{5 n}$ and $\mathbf{u}(t):=$ $\left(\mathbf{u}_{1}(t), \mathbf{u}_{2}(t), \ldots, \mathbf{u}_{n}(t)\right) \in \mathbb{R}^{2 n}$. Then we have the following initial-value problem for $\mathscr{A}_{i}$ :

$$
\begin{aligned}
\dot{\mathbf{x}} & =\mathbf{f}(\mathbf{x})+\mathbf{B u}(t), \\
\mathbf{x}\left(t_{0}\right) & :=\mathbf{x}_{0}, \quad t_{0} \geq 0,
\end{aligned}
$$

where if $\mathbf{0}$ is a $5 \times 2$ matrix of all zero entries,

$$
\mathbf{B}=\left[\begin{array}{cccc}
\mathbf{B}_{1} & \mathbf{0} & \cdots & \mathbf{0} \\
\mathbf{0} & \mathbf{B}_{2} & \cdots & \mathbf{0} \\
\vdots & \vdots & \ddots & \vdots \\
\mathbf{0} & \mathbf{0} & \cdots & \mathbf{B}_{n}
\end{array}\right] .
$$

Now, assume that the final position of $\mathscr{A}_{i}$ is at the point $\left(x_{i}, y_{i}\right)=\left(p_{i 1}, p_{i 2}\right)$ and final orientation at this point is $\theta_{i}=$ $p_{i 3}$. Its final instantaneous velocity vector is $\left(v_{i}, \omega_{i}\right)=(0,0)$. Then it is clear that the points

$$
\mathbf{x}_{i}^{*}:=\left(p_{i 1}, p_{i 2}, p_{i 3}, 0,0\right) \in \mathbb{R}^{5}
$$

are the components of the equilibrium point of system (5) in which we are interested; that is,

$$
\mathbf{x}_{e}:=\left(\mathbf{x}_{1}^{*}, \mathbf{x}_{2}^{*}, \ldots, \mathbf{x}_{n}^{*}\right) \in \mathbb{R}^{5 n}
$$

2.1. Leader-Follower Based Formation Scheme. Next we define two reference frames: the body frame which is fixed with the rotating body of the leader, $\mathscr{A}_{1}$, and a space frame, the inertial frame similar to one proposed in [16].

We assign a Cartesian coordinate system $(X-Y)$ fixed on the leader body, as shown in Figure 2 adopted from [27], based on the concept of an instantaneous corotating frame of reference. Thus, when the leader $\mathscr{A}_{1}$ rotates, we have a rotation of the $X-Y$-axes.

To define the corotating frame of reference, first an origin is selected on the leader robot at $\left(x_{1}, y_{1}\right)$. An axis of rotation is then set up, which is perpendicular to the plane of motion of the leader. Thus, at any selected moment $t$, the chosen rotating frame of reference rotates at an angular rate equal to the rate of rotation of the leader $\mathscr{A}_{1}$ about $\left(x_{1}, y_{1}\right)$. Let $r_{1 k}$ represent the straight-line distance between the reference point of the leader and the $k$ th follower, and $\alpha_{1 k}$ represents the angle measured between the straight line joining the reference points of the leader and the $k$ th follower and $X$ axis. Thus, given the leader's position and orientation, as long as $\left(r_{1 k}, \alpha_{1 k}\right)$, as shown in Figure 2, is fixed, the $k$ th follower robot's position will be unique. We define the shape of the formation of the mobile robots as $\zeta=\left[\zeta_{12}, \zeta_{13}, \ldots, \zeta_{1 n}\right]^{T}$, where $\zeta_{1 k}=\left[r_{1 k}, \alpha_{1 k}\right]^{T}$ for $k \in\{2, \ldots, n\}$. 


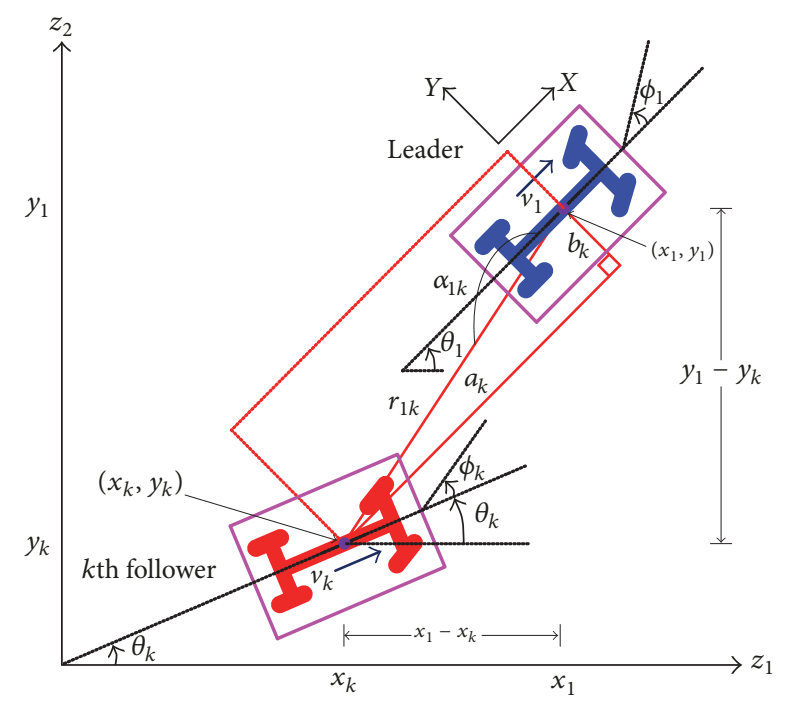

FIGURE 2: The proposed scheme utilizing a rotation of axes with the body frame fixed at the leader robot.

Definition 1. Let $2 r_{k}<r_{1 k}^{d}<2 r_{k}+\varsigma$, where, for $\varsigma>0$ and $k \in\{2, \ldots, n\}$, the group of mobile robots make a $\zeta^{d}=$ $\left[\zeta_{12}^{d}, \zeta_{13}^{d}, \ldots, \zeta_{1 n}^{d}\right]^{T}$ formation, if $\exists T>0, \forall t>T$ :

$$
\begin{aligned}
& r_{1 k}=r_{1 k}^{d}, \\
& \alpha_{1 k}=\alpha_{1 k}^{d} .
\end{aligned}
$$

This gives then the polar coordinate representation of the follower's position relative to that of the leader. However, such representations using polar coordinates lead to certain singularities in the controllers [16]. To avoid such singular points, we consider the position of the $k$ th follower by considering the relative distances of the $k$ th follower from the leader $\mathscr{A}_{1}$ along the given $X$ and $Y$ directions. Thus, we have

$$
\begin{gathered}
A_{k}=-\left(x_{1}-x_{k}\right) \cos \theta_{1}-\left(y_{1}-y_{k}\right) \sin \theta_{1}, \\
B_{k}=\left(x_{1}-x_{k}\right) \sin \theta_{1}-\left(y_{1}-y_{k}\right) \cos \theta_{1},
\end{gathered}
$$

for $k \in\{2, \ldots, n\}$, and $A_{k}$ and $B_{k}$ are the $k$ th follower relative positions with respect to the $X-Y$ coordinate system. If $A_{k}$ and $B_{k}$ are known and fixed, the follower's position will be distinct. Thus, to obtain a desired formation, one needs to know the design parameters $a_{k}$ and $b_{k}$ and the desired relative positions along the $X-Y$ directions, such that the control objective would be to achieve $A_{k} \rightarrow a_{k}$ and $B_{k} \rightarrow b_{k}$. That is, $r_{1 k} \rightarrow r_{1 k}^{d}$ and $\alpha_{1 k} \rightarrow \alpha_{1 k}^{d}$, where $r_{1 k}^{d}=\sqrt{a_{k}^{2}+b_{k}^{2}}$ and $\alpha_{1 k}^{d}=$ $\tan \left(a_{k} / b_{k}\right)$, hence maintaining local rigidity of any formation.

Definition 2. We define a locally rigid formation as one which is maintained but allows for slight changes or distortions in the distances and angles between any two vehicles temporarily, while enroute to the target and task completion.

Remark 3 (formation initialization). Prescribed formations can be initialized by assigning appropriately the design parameters $a_{k}$ and $b_{k}$. This will initiate the vehicles from their arbitrary initial positions to move into a desired formation pattern.

2.2. Formation Control Objective. Design accelerationbased control laws $\left(\sigma_{i 1}, \sigma_{i 2}\right)$ which are functions of $\mathbf{x}_{i}:=$ $\left(x_{i}, y_{i}, \theta_{i}, v_{i}, \omega_{i}\right) \in \mathbb{R}^{5}$ for $i \in\{1, \ldots, n\}$, for each vehicle such that the C-ITS in a prescribed formation moves towards a predefined target of the leader in an obstacle cluttered environment. In addition, we also want the C-ITS to ensure obstacle and collision avoidance and maintain a prescribed locally rigid formation.

\section{Artificial Potential Field Function}

This section formulates collision-free trajectories of the vehicle system under kinodynamic constraints. We want to design the acceleration controllers, $\sigma_{i 1}$ and $\sigma_{i 2}$, so that the team of vehicles moves safely towards the target of the leader while maintaining a prescribed locally rigid formation. We will design the attractive potential field functions which establish and translate formation and the repulsive potential field functions which ensure collision and obstacle avoidances.

In the following subsections, we will design these attractive and repulsive potential field functions which are subsequently summed to form the total potentials or artificial potential field of the system.

\subsection{Attractive Potential Field Functions}

3.1.1. Attraction to Target. A target is assigned to the leader. When the leader moves towards its defined target, the follower vehicles move with the leader, maintaining the formation. We want the leader $\mathscr{A}_{1}$ to start from an initial position, move towards a target, and finally converge at the center of the target.

Definition 4. The stationary target for the leader robot $\mathscr{A}_{1}$ is a circular disk with center $\left(p_{11}, p_{12}\right)$ and radius $r t$.

The leader $\mathscr{A}_{1}$ will move towards its defined target, while the follower vehicles move with their leader maintaining a desired relative position, hence a locally rigid formation. For the attraction of $\mathscr{A}_{1}$ to its target, we consider an attractive potential function:

$$
V_{1}(\mathbf{x})=\frac{1}{2}\left[\left(x_{1}-p_{11}\right)^{2}+\left(y_{1}-p_{12}\right)^{2}+v_{1}^{2}+\omega_{1}^{2}\right] .
$$

The above function is a measure of its convergence to the target with the inclusion of the velocity components [14]. For the follower vehicle $\mathscr{A}_{i}$ for $i=2, \ldots, n$ to maintain its desired relative position with respect to the leader, $\mathscr{A}_{1}$, we utilize the following potential function:

$$
V_{i}(\mathbf{x})=\frac{1}{2}\left[\left(A_{i}-a_{i}\right)^{2}+\left(B_{i}-b_{i}\right)^{2}+v_{i}^{2}+\omega_{i}^{2}\right],
$$

for $i=2, \ldots, n$, where $a_{i}$ and $b_{i}$ will have unique values as dictated by the shape of the desired formation. 
3.1.2. Auxiliary Function. To guarantee the convergence of the vehicles to their designated targets, we design an auxiliary function as

$$
\begin{aligned}
& G_{1}(\mathbf{x}) \\
& \quad=\frac{1}{2}\left[\left(x_{1}-p_{11}\right)^{2}+\left(y_{1}-p_{12}\right)^{2}+\rho_{1}\left(\theta_{1}-p_{13}\right)^{2}\right],
\end{aligned}
$$

where $p_{13}$ is the prescribed final orientation of the leader robot, $\mathscr{A}_{1}$, and

$$
G_{i}(\mathbf{x})=\frac{1}{2}\left[\left(A_{i}-a_{i}\right)^{2}+\left(B_{i}-b_{i}\right)^{2}+\rho_{i}\left(\theta_{i}-\theta_{1}\right)^{2}\right],
$$

for $i=2, \ldots, n$. The function ensures that the controllers become zero at the leader's target. The constant $\rho_{i}$ is a binary constant denoted in (13) and (14) as the angle-gain parameter for $\theta_{i}, i=1, \ldots, n$. An angle-gain parameter will take a value of one only if a final predefined orientation is warranted; else it takes the default value of zero. This auxiliary function is then multiplied to the repulsive potential field functions.

3.2. Repulsive Potential Field Functions. We desire each vehicle to avoid all fixed and moving obstacles intersecting its path. Hence, we construct appropriate obstacle avoidance functions that measure the Euclidean distances between mobile robots and the obstacles on our roads. To obtain the desired avoidance, we generate repulsive potential field around the obstacles by designing a repulsive potential field function for each obstacle in accordance with LbCS. The repulsive potential fields function is an inverse function that encodes an avoidance function to the denominator and a control parameter in the numerator [14].

3.2.1. Fixed Obstacles. Let us fix $n \in \mathbb{N}$ solid obstacles on the roads, such as potholes, distance marker posts, or road hazard delineators. We assume that the $l$ th obstacle is a circular disk with center $\left(o_{l 1}, o_{l 2}\right)$ and radius $r o_{l}$. For the $i$ th vehicle to avoid the $l$ th obstacle, we consider an avoidance function:

$$
\mathrm{FO}_{i l}(\mathbf{x})=\frac{1}{2}\left[\left(x_{i}-o_{l 1}\right)^{2}+\left(y_{i}-o_{l 2}\right)^{2}-\left(r o_{l}+r_{i}\right)^{2}\right],
$$

where $i=1, \ldots, n$ and $l=1, \ldots, q$.

Consider, for example, the presence of three obstacles (i.e., $q=3$ ) within the workspace, with $0<z_{1}<70$ and $0<z_{2}<70$. The total potentials, where $\alpha_{1 l}>0$ is a control or tuning parameter, which govern the motion of the leader $\mathscr{A}_{1}$ are

$$
V_{1}(\mathbf{x})+\sum_{l=1}^{3} \frac{\alpha_{1 l}}{\mathrm{FO}_{1 l}(\mathbf{x})}
$$

Figure 3 presents a three-dimensional view of the total potentials and Figure 4 presents the corresponding contour plot produced by (16).

The total potentials are generated for target attraction and avoidance of three stationary disk-shaped obstacles. For better visualization, the target of the leader is located at $\left(p_{1}, p_{2}\right)=(35,35)$, and the disks are fixed at

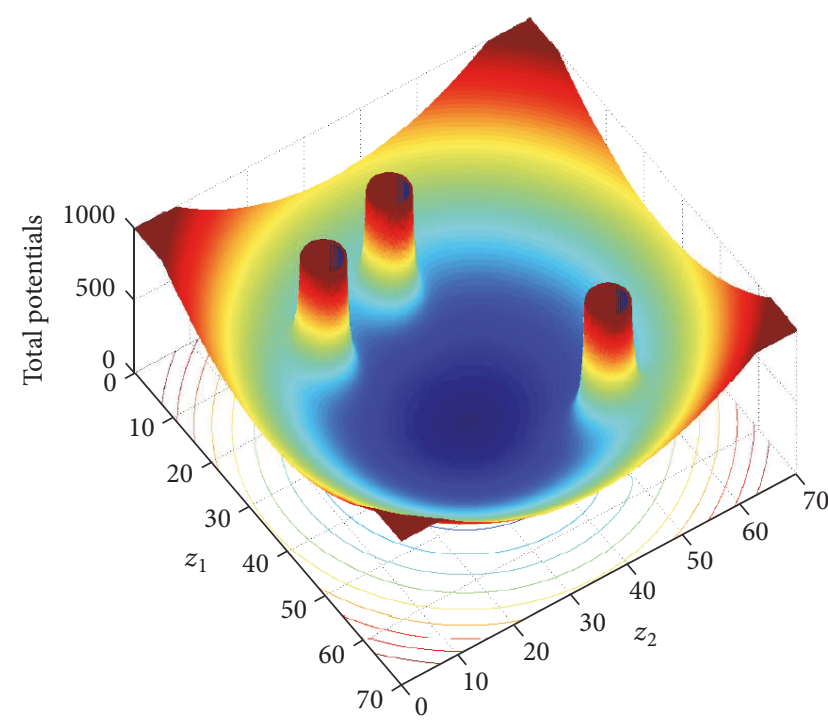

Figure 3: A three-dimensional view of the total potentials.

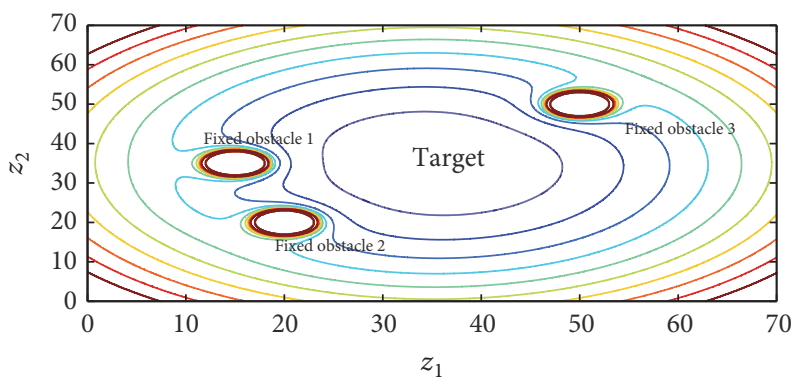

FIGURE 4: The contour plot of the total potential.

$\left(o_{11}, o_{12}\right)=(15,35),\left(o_{21}, o_{22}\right)=(20,20)$, and $\left(o_{31}, o_{32}\right)=$ $(50,50)$, with radii of $r o_{l}=3$, while $\alpha_{l 1}=1000$, for $l=$ $1, \ldots, 3$. Also, the velocity and angular components of the lead vehicle have been treated as constants $\left(v_{1}=0.5, \omega_{1}=0\right.$, and $\left.\theta_{1}=0\right)$.

\subsection{Boulevard Limitations}

Definition 5. Consider a section of the road defined, for some $\eta_{1}>2 r_{i}$, for $i=1, \ldots, n$, as

$$
W S=\left\{\left(z_{1}, z_{2}\right) \in \mathbb{R}^{2}: 0 \leq z_{2} \leq \eta_{1}\right\}
$$

The boundaries of the road section, illustrated in Figure 5, are defined as follows:

(a) Lower boundary: $B_{1}=\left\{\left(z_{1}, z_{2}\right) \in \mathbb{R}^{2}: z_{2}=0\right\}$

(b) Upper boundary: $B_{2}=\left\{\left(z_{1}, z_{2}\right) \in \mathbb{R}^{2}: z_{2}=\eta_{1}\right\}$

We require the prescribed formation to stay within the boundaries of the road at all time $t \geq 0$. In our LbCS, these boundaries are considered as fixed obstacles. For the $i$ th robot 


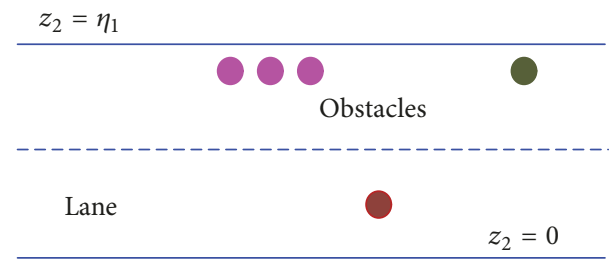

FIGURE 5: A schematic representation of the road with obstacles.

to avoid these, we define the following potential functions for the upper and lower boundaries, respectively:

$$
\begin{aligned}
& W_{i 1}(\mathbf{x})=y_{i}-r_{i}, \\
& W_{i 2}(\mathbf{x})=\eta_{1}-\left(y_{i}+r_{i}\right) .
\end{aligned}
$$

Now, since $\eta_{1}>2 r_{i}$, each of the functions is positive in WS, for $i=1, \ldots, n$. As discussed before, these obstacle avoidance functions will be combined with appropriate tuning parameters to generate repulsive potential field functions for the boundaries of the workspace.

3.4. Final Orientation of Formation. Although the final position is reachable, it is virtually impossible to simultaneously harvest exact orientations via continuous feedback controllers of nonholonomic systems [14], a direct result of Brockett's theorem [28]. We utilize the concepts of ghost wall and minimum distance technique (MDT) from [14] to force final orientations of the vehicles. This is needed to confine the vehicles to within the lane markings and later for parking a vehicle into a parking bay. To begin, we construct ghost walls along the three sides of the final positions of the vehicles, treated as a parking bay, with the orientation depending on the desired final orientation of the vehicles. To avoid the ghost walls of each vehicle's parking bay, we utilize MDT, which gives the perpendicular distance between the closest point on each $k$ th ghost wall and $\left(x_{i}, y_{i}\right)$. The avoidance of these closet points on a given ghost wall at any time $t \geq 0$ essentially results in the avoidance of the entire wall by a vehicle.

Now let us consider the $k$ th ghost wall in the $z_{1} z_{2}$-plane from the point $\left(a_{k 1}, b_{k 1}\right)$ to the point $\left(a_{k 2}, b_{k 2}\right)$. We assume that the point $\left(x_{i}, y_{i}\right)$ is closest to it at the tangent line which passes through the point. From geometry, it is known that if $\left(L x_{i k}, L y_{i k}\right)$ is the point of intersection of this tangent, then

$$
\begin{aligned}
& L x_{i k}=a_{k 1}+\lambda_{i k}\left(a_{k 2}-a_{k 1}\right), \\
& L y_{i k}=b_{k 1}+\lambda_{i k}\left(b_{k 2}-b_{k 1}\right),
\end{aligned}
$$

where

$$
\begin{aligned}
& \lambda_{i k}=\left(x_{i}-a_{k 1}\right) d_{k}+\left(y_{i}-b_{k 1}\right) r_{k}, \\
& d_{k}=\frac{a_{k 2}-a_{k 1}}{\left(a_{k 2}-a_{k 1}\right)^{2}+\left(b_{k 2}-b_{k 1}\right)^{2}}, \\
& r_{k}=\frac{b_{k 2}-b_{k 1}}{\left(a_{k 2}-a_{k 1}\right)^{2}+\left(b_{k 2}-b_{k 1}\right)^{2}} .
\end{aligned}
$$

If $\lambda_{i k} \geq 1$, then we let $\lambda_{i k}=1$; if $\lambda_{i k} \leq 0$, then we let $\lambda_{i k}=0$; otherwise we accept the value of $\lambda_{i k}$ between 0 and 1 , in which case there is a perpendicular line to the point $\left(L x_{i k}, L y_{i k}\right)$ on the ghost wall from the center $\left(x_{i}, y_{i}\right)$ of the $i$ th vehicle at every time $t \geq 0$.

The leader will be avoiding the 1st, 2nd, and 3rd ghost walls, while the $i$ th follower will be avoiding the $(3 i-2),(3 i-$ 1 ), and ( $3 i$ ) ghost walls for $i=2, \ldots, n$. Now, for the $i$ th vehicle to avoid the closest point of each of the $k$ th line segments, we consider an avoidance function

$$
L S_{i k}(\mathbf{x})=\frac{1}{2}\left[\left(x_{i}-L x_{i k}\right)^{2}+\left(y_{i}-L y_{i k}\right)^{2}-r_{i}^{2}\right],
$$

for $k \in\{3 i-2,3 i-1,3 i\}$ and $i=1, \ldots, n$.

3.5. Moving Obstacles. To generate feasible trajectories, we consider moving obstacles in the workspace, in which the C-ITS has prior knowledge. Here, each vehicle has to be treated as a moving obstacle for all other vehicles on the road. The vehicles will have to travel towards their targets while avoiding another vehicle in their path. For the vehicle $\mathscr{A}_{i}$ to avoid the vehicle $\mathscr{A}_{j}$, via vehicle-to-vehicle (V-V) communication, we adopt an avoidance function:

$$
\begin{aligned}
\mathrm{MO}_{i j}(\mathbf{x})=\frac{1}{2}\left[\left(x_{i}-x_{j}\right)^{2}+\left(y_{i}-y_{j}\right)^{2}-\left(r_{i}\right)^{2}\right], & \\
& \text { for } i, j=1, \ldots, n \text { with } i \neq j .
\end{aligned}
$$

3.5.1. Dynamic Constraints. Practically, the steering angle of the $i$ th autonomous vehicle is limited due to mechanical singularities, while the translational speed is restricted due to safety reasons [14]. Subsequently, we have the following;

(i) $\left|v_{i}\right|<v_{\max }$, where $v_{\max }$ is the maximal achievable speed of the $i$ th vehicle.

(ii) $\left|\omega_{i}\right|<v_{\max } /\left|\rho_{\min }\right|$, where $\rho_{\min }:=L_{i} / \tan \left(\phi_{\max }\right)$. This condition arises due to the boundedness of the steering angle $\phi_{i}$. That is, $\left|\phi_{i}\right| \leq \phi_{\max }<\pi / 2$, where $\phi_{\max }$ is the maximal steering angle.

Remark 6. For simplicity, the values of $v_{\max }$ and $\phi_{\max }$ will be kept the same for each vehicle.

As per the LbCS, for each dynamic constraint, we design a corresponding artificial obstacle. For example, we consider the artificial obstacle $\mathrm{AO}_{i}=\left\{v_{i} \in \mathbb{R}: v_{i} \leq-v_{\max }\right.$ or $v_{i} \geq$ $\left.v_{\max }\right\}$ for the constraint tagged to $v_{i}$. We can create similar artificial obstacles for the other limitations as well. Hence, we consider the following avoidance functions:

$$
\begin{aligned}
& U_{i 1}(\mathbf{x})=\frac{1}{2}\left(v_{\max }-v_{i}\right)\left(v_{\max }+v_{i}\right), \\
& U_{i 2}(\mathbf{x})=\frac{1}{2}\left(\frac{v_{\max }}{\left|\rho_{\min }\right|}-\omega_{i}\right)\left(\frac{v_{\max }}{\left|\rho_{\min }\right|}+\omega_{i}\right),
\end{aligned}
$$




\section{Design of the Acceleration-Based Controllers}

4.1. Lyapunov Function. We now construct the total potentials, that is, a Lyapunov function for system (1). First, for $i=1, \ldots, n$, we introduce the following control parameters that we will use in the repulsive potential functions:

(i) $\alpha_{i l}>0, l=1, \ldots, q$, for the collision avoidance of $q$ disk-shaped obstacles.

(ii) $\beta_{i s}>0, s=1,2$, for the avoidance of the artificial obstacles from dynamic constraints.

(iii) $\eta_{i j}>0, j=1, \ldots, n, i \neq j$, for the collision avoidance between any two vehicles.

(iv) $\kappa_{i p}>0, p=1, \ldots, 2$, for the avoidance of the lane boundaries.

(v) $\gamma_{i k}>0, k=1, \ldots, 3 n$, for the avoidance of the parking bays.

The above parameters are determined via heuristics, but there is also an opportunity to obtain optimal values through optimization techniques. Using these, we now construct the following Lyapunov function for system (1) with two components, namely, the attractive and repulsive potential field functions:

$$
\begin{aligned}
& L_{(1)}(\mathbf{x})=\sum_{i=1}^{n}\left[V_{i}(\mathbf{x})+G_{i}(\mathbf{x})\right. \\
& \left.\cdot\left(\sum_{l=1}^{q} \frac{\alpha_{i l}}{\mathrm{FO}_{i l}(\mathbf{x})}+\sum_{s=1}^{2} \frac{\beta_{i s}}{U_{i s}(\mathbf{x})}+\sum_{p=1}^{2} \frac{\kappa_{i p}}{W_{i p}(\mathbf{x})}\right)\right] \\
& +\sum_{i=1}^{n} G_{i}(\mathbf{x})\left(\sum_{\substack{j=1 \\
j \neq i}}^{n} \frac{\eta_{i j}}{\mathrm{MO}_{i j}(\mathbf{x})}+\sum_{k=1}^{3 n} \frac{\gamma_{i k}}{L S_{i k}(\mathbf{x})}\right) .
\end{aligned}
$$

4.2. Nonlinear Acceleration Controllers. The process of designing the feedback controllers begins by noting that the functions $f_{i k}$ to $g_{i j}$ for $i=1, \ldots, n, j=1,2$, and $k=1, \ldots, 3$ are defined as in the Appendix (on suppressing $\mathbf{x}$ ).

Remark 7. The choice of total potential $L(\mathbf{x})$ given in (24) with its terms specified in (11)-(16), (18a), (18b), and (21)-(23) has the following properties:

(i) It attains a minimum value when the robots are at their desired locations.

(ii) It goes to infinity whenever one or more robots come in contact with an obstacle.

Remark 8. With the interrobot bounds (see (12) and (22)) in place, it is guaranteed that the robots reestablish the predetermined formation if the robot positions are slightly distorted with the encounter of obstacle(s) soon after the avoidance and before reaching the target.

So, we design the following theorem.
Theorem 9. Consider $n$ car-like mobile robots in the C-ITS, whose motion is governed by the ODEs described in system (1). The principal goal is to establish and control a prescribed formation, facilitate maneuvers of the vehicles within a constrained environment, and reach the target configuration while maintaining a desired formation. The subtasks include restrictions placed on the workspace, convergence to predefined targets, and consideration of kinodynamic constraints. Utilizing the attractive and repulsive potential field functions, the following continuous time-invariant acceleration control laws can be generated in accordance with the LbCS of system (1):

$$
\begin{aligned}
\sigma_{i 1} & =-\frac{\left[\delta_{i 1} v_{i}+f_{i 1} \cos \theta_{i}+f_{i 2} \sin \theta_{i}\right]}{g_{i 1}}, \\
\sigma_{i 2} & =-\frac{\left[\delta_{i 2} \omega_{i}+\left(L_{i} / 2\right)\left(f_{i 2} \cos \theta_{i}-f_{i 1} \sin \theta_{i}\right)+f_{i 3}\right]}{g_{i 2}},
\end{aligned}
$$

for $i=1, \ldots, n$, where $\delta_{i 1}>0$ and $\delta_{i 2}>0$ are constants commonly known as convergence parameters.

\section{Stability Analysis}

We utilize Lyapunov's direct method to provide a mathematical proof of stability of system (1).

Theorem 10. Let $\left(p_{11}, p_{12}\right)$ be the position of the target of the leader and let $p_{i 3}$, for $i=1, \ldots, n$, be the prescribed final orientations of the robots. Let $p_{i 1}$ and $p_{i 2}$ satisfy

$$
\begin{aligned}
& a_{i}=-\left(p_{11}-p_{i 1}\right) \cos \theta_{1}-\left(p_{12}-p_{i 2}\right) \sin \theta_{1}, \\
& b_{i}=\left(p_{11}-p_{i 1}\right) \sin \theta_{1}-\left(p_{12}-p_{i 2}\right) \cos \theta_{1},
\end{aligned}
$$

for any given $a_{i}$ and $b_{i}$, for $i=2, \ldots, n$. If $\mathbf{x}_{e} \in \mathbb{R}^{5 n}$ as defined in (8) is an equilibrium point for (1), then $\mathbf{x}_{e} \in D\left(L_{(1)}(\mathbf{x})\right)$ is a stable equilibrium point of system (1).

Proof. One can easily verify the following, for $i \in\{1, \ldots, n\}$ :

(1) $L_{(1)}(\mathbf{x})$ is defined as continuous and positive over the domain $D\left(L_{(1)}(\mathbf{x})\right)=\left\{\mathbf{x} \in \mathbb{R}^{5 n}: \mathrm{FO}_{i l}(\mathbf{x})>0, l=\right.$ $1, \ldots, q ; \mathrm{MO}_{i j}(\mathbf{x})>0, j=1, \ldots, n, j \neq i ; W_{i p}(\mathbf{x})>$ $0, p=1, \ldots, 4 ; L S_{i k}(\mathbf{x})>0, k=1, \ldots, 3 n ; U_{i s}(\mathbf{x})>$ $0, s=1,2\}$.

(2) $L_{(1)}\left(\mathbf{x}_{e}\right)=0 ; \dot{L}_{(1)}\left(\mathbf{x}_{e}\right)=0$.

(3) $L_{(1)}(\mathbf{x})>0 \forall \mathbf{x} \in D\left(L_{(1)}(\mathbf{x})\right) / \mathbf{x}_{e}$.

(4) $\dot{L}_{(1)}(\mathbf{x})=-\sum_{i=1}^{n}\left(\delta_{i 1} v_{i}^{2}+\delta_{i 2} \omega_{i}^{2}\right) \leq 0, \forall \mathbf{x} \in D\left(L_{(1)}(\mathbf{x})\right)$.

(5) $L_{(1)}(\mathbf{x}) \in C^{1}\left(D\left(L_{(1)}(\mathbf{x})\right)\right)$.

Hence, $L_{(1)}(\mathbf{x})$ is classified as a Lyapunov function for system (1) and $\mathbf{x}_{e}$ is a stable equilibrium point in the sense of Lyapunov.

\section{Simulation Results}

In this section, we illustrate the effectiveness of the proposed continuous controllers by simulating a number of virtual scenarios for the C-ITS on our roads. 


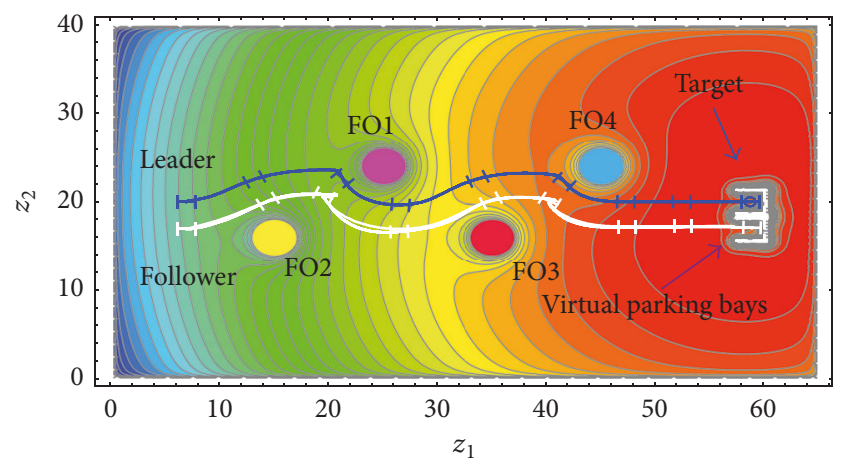

FO: Fixed obstacle

FIgURE 6: The evolution of C-ITS trajectories and the contour plot in the presence of obstacles in Scenario 1.

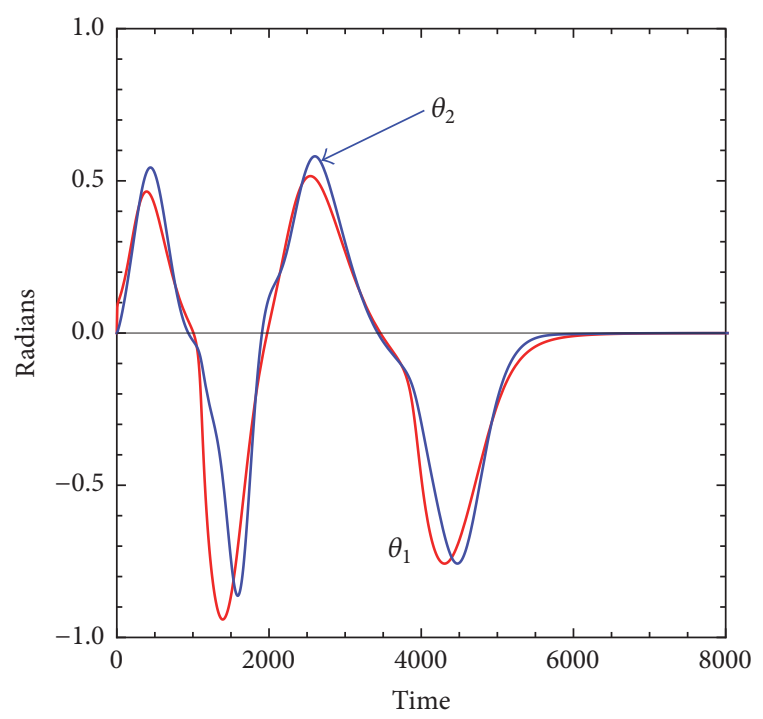

Figure 7: Orientations of $\mathscr{A}_{1}$ and $\mathscr{A}_{2}$ for Scenario 1.

6.1. Scenario 1: Line Formation in the Presence of Obstacles. We consider the motion of two automated vehicles in a line formation in the presence of obstacles. The follower vehicle is prescribed a position relative to the leader $\mathscr{A}_{1}$ as seen in Figure 6. While the leader moves towards its target, the follower maintains a desired distance and orientation relative to the leader, therefore maintaining a locally rigid formation throughout the journey.

Assuming that the appropriate units have been accounted for, Table 1 provides the corresponding initial and final configurations of the two vehicles and other parameters required to simulate Scenario 1.

Figure 6 also depicts the contour plot of the potential fields of the system enroute to the final destination. Figures 7 and 8 show the orientations and velocities of $\mathscr{A}_{1}$ and $\mathscr{A}_{2}$, respectively. Figure 9 depicts the time evolution of the nonlinear controllers of the leader $\mathscr{A}_{1}$, and Figure 10 compares the relative distance $r_{12}$ to the desired relative distance $r_{12}^{d}$. It is evident that the formation is slightly distorted when the

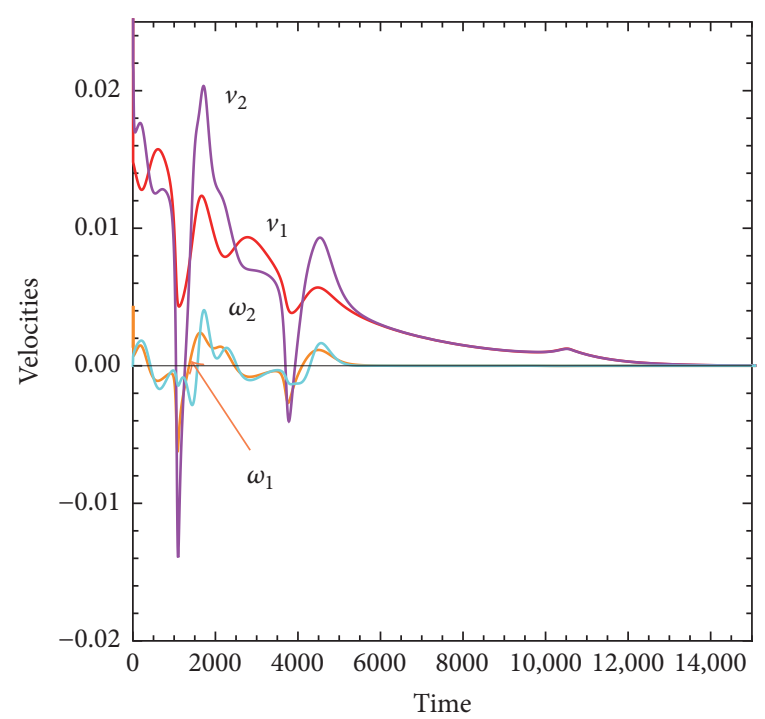

FIGURE 8: Translational and rotational velocities for Scenario 1.

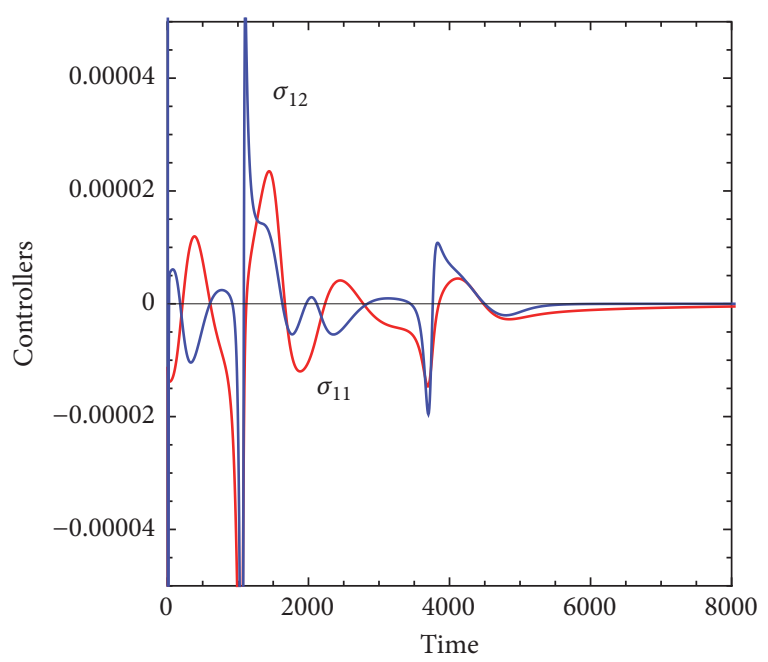

Figure 9: Accelerations $\sigma_{11}$ and $\sigma_{12}$ of $\mathscr{A}_{1}$ for Scenario 1.

pair of cooperative cars encounter an obstacle; however, the desired formation is reestablished before the pair reach the final destination.

6.2. Scenario 2: Effect of Noise on Line Formation. To evaluate the robustness of the proposed scheme, we look at the effect of noise on the formation of the C-ITS. It is sufficient to include the noise parameters in the components $A_{k}$ and $B_{k}$ which define the follower vehicles' relative position to the leader vehicle, with respect to the $X-Y$ coordinate system, similar to the one proposed in [29]. Thus we have

$$
\begin{gathered}
A_{k}=-\left(x_{1}-x_{k}\right) \cos \theta_{1}-\left(y_{1}-y_{k}\right) \sin \theta_{1}+\xi \gamma_{k}(t), \\
B_{k}=\left(x_{1}-x_{k}\right) \sin \theta_{1}-\left(y_{1}-y_{k}\right) \cos \theta_{1}+\xi \nu_{k}(t) .
\end{gathered}
$$

The terms $\xi \gamma_{k}(t)$ and $\xi \nu_{k}(t)$ are the small disturbances, where $\xi \in[0,1]$ is the noise level, while $\gamma_{k}(t)$ and $\nu_{k}(t)$ are randomized time-dependent variables such that $\gamma_{k}(t) \in[-1,1]$ 
TABLE 1: Numerical values of initial and final states, constraints, and parameters for Scenario 1.

\begin{tabular}{ll}
\hline & Initial configuration \\
\hline Rectangular positions & $\left(x_{1}, y_{1}\right)=(7,20)$ and $\left(x_{2}, y_{2}\right)=(7,17)$ \\
Velocities & $v_{i}=0.5$ and $\omega_{i}=0$, for $i=1,2$ \\
Angular positions & $\theta_{i}=0$, for $i=1,2$ \\
& $\left(a_{11}, b_{11}\right)=(57.5,21.3)$ and $\left(a_{12}, b_{12}\right)=(60.1,21.3)$ \\
Parking bays & $\left(a_{31}, b_{31}\right)=(57.5,18.7)$ and $\left(a_{32}, b_{32}\right)=(60.1,18.7)$ \\
& $\left(a_{41}, b_{41}\right)=(57.5,18.2)$ and $\left(a_{42}, b_{42}\right)=(60.1,18.2)$ \\
& $\left(a_{61}, b_{61}\right)=(57.5,15.6)$ and $\left(a_{62}, b_{62}\right)=(60.1,15.6)$ \\
\hline Dimensions of robots & Constraints and parameters \\
Leader's target & $L_{i}=1.6$ and $l_{i}=1.2$ for $i=1,2$ \\
Final orientations & $\left(p_{11}, p_{12}\right)=(57,20) ; r t=0.5$ \\
\hline Max. translational velocity & $p_{13}=p_{23}=0$ \\
Max. steering angle & $v_{\max }=5$ \\
Clearance parameters & $\phi_{\max }=\frac{\pi}{2}$ \\
Fixed obstacles & $\epsilon_{1}=0.1$ and $\epsilon_{2}=0.05$ \\
& $\left(o_{11}, o_{12}\right)=(15,16),\left(o_{21}, o_{22}\right)=(25,24)$, and $\left(o_{31}, o_{32}\right)=(35,16)$ \\
& $\left(o_{41}, o_{42}\right)=(45,24)$ and $r o_{l}=2$ for $l=2, \ldots, 4$ \\
\hline & Control and convergence parameters \\
Collision avoidance & $\eta_{i j}=0.001$, for $i, j=1,2, j \neq i$ \\
& $\kappa_{i p}=0.1$, for $i=1,2, p=1, \ldots, 2$ \\
& $\gamma_{i k}=0.1$, for $i=1,2, k=1, \ldots, 3 n$ \\
Dynamics constraints & $\alpha_{11}=1, \alpha_{12}=\alpha_{14}=0.1$, and $\alpha_{13}=2$ \\
Convergence & $\alpha_{2 l}=0.01$ for $l=1, \ldots, 4$ \\
& $\beta_{i s}=0.01$, for $i, s=1,2$ \\
& $\delta_{11}=3000, \delta_{12}=100, \delta_{21}=10$, and $\delta_{22}=100$ \\
\hline
\end{tabular}

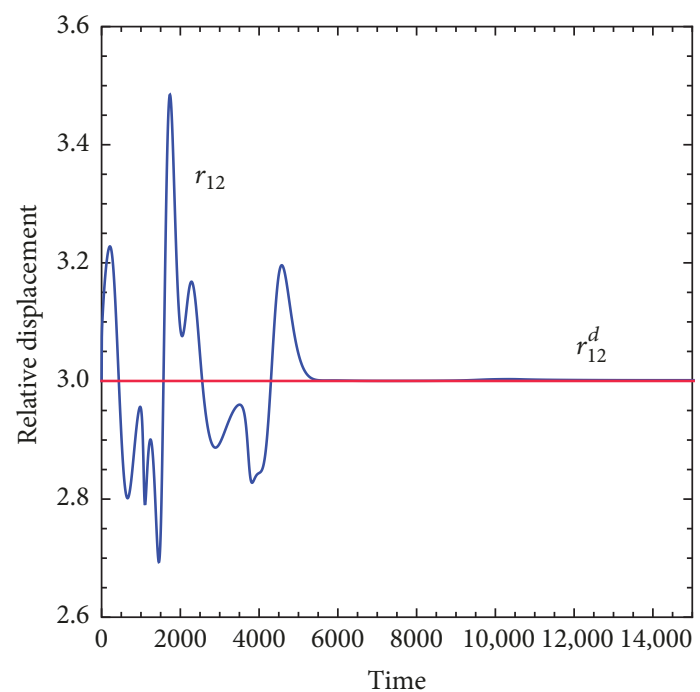

FIGURE 10: The relative distance of the follower to the leader vehicle compared to the desired relative distance in Scenario 1.

and $v_{k}(t) \in[-1,1]$. Figure 11 shows the trajectories and the control signals under the influence of small disturbances, $\xi \in$ $[0,1]$. It is observed that the pair of cooperative cars maintain the line formation throughout their road journey even under the influence of the noise terms. There are slight distortions in their formation when the pair of cooperative vehicles encounter obstacles but these distortions are temporary. Also, the disturbances in the controllers at $t=11,000$ units are a result of the parking bays. The two cars align themselves to achieve the desired orientations inside the parking bays. The final orientations are forced as a result of the repulsive potentials created by the walls of the parking bays.

6.3. Scenario 3: Diamond Formation. In this scenario, we have considered the leader vehicle at the center of a Diamond Formation with the followers positioned at each vertex (see Figure 12). The figure shows the formation maneuvered from an initial state to a predefined final state, with collision and obstacle avoidance.

Figure 12 also depicts the contour plot of the potential fields and the corresponding collision-free path over the defined workspace, $0<z_{1}<40$, containing the obstacles on the road. Figures 13 and 14 depict the time evolution of the nonlinear controllers of the leader and its follower vehicles. Assuming that the appropriate units have been accounted for, Table 2 (if different from Table 1) provides the corresponding initial and final configurations of the 5-car C-ITS and other parameters required to simulate Scenario 3. The coordinates of the parking bays can be obtained from Figure 12.

Clearly the translational and rotational accelerations of the vehicles decrease as the formation approaches a fixed obstacle and increase once it is able to evade it. Moreover, Figures 15 and 16 compare the relative distance $A_{k}$ to the 


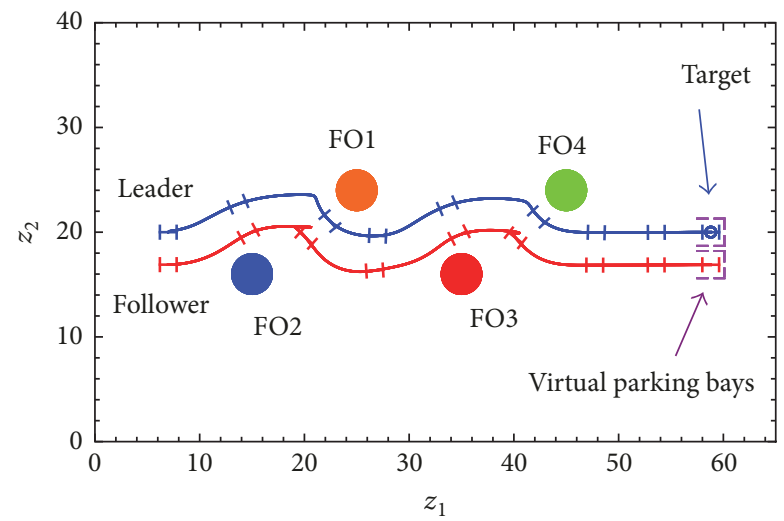

FO: Fixed obstacle

(a) Trajectories for $\xi=0.1$

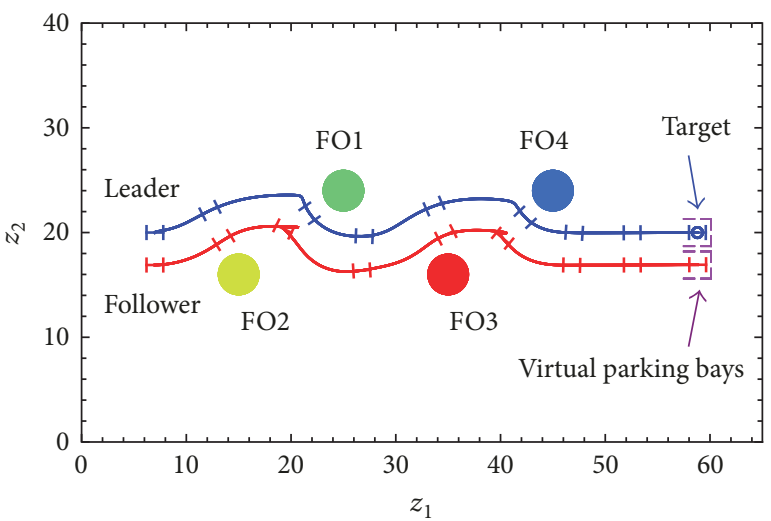

FO: Fixed obstacle

(c) Trajectories for $\xi=0.2$

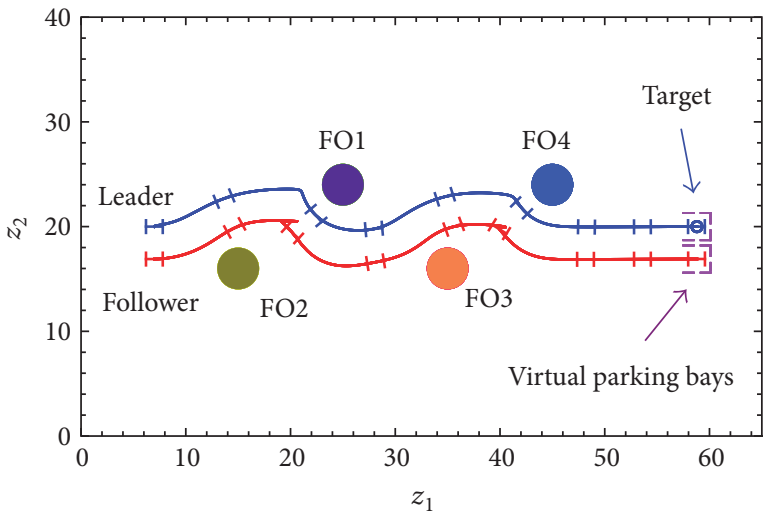

FO: Fixed obstacle

(e) Trajectories for $\xi=0.5$

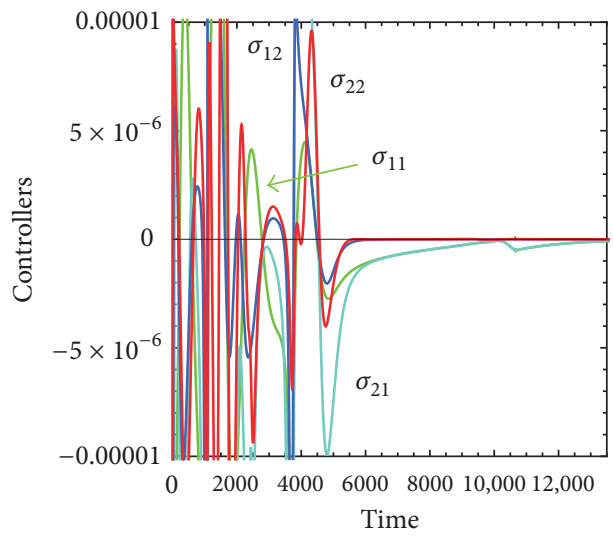

(b) Control signals, $\sigma_{i 1}$ and $\sigma_{i 2}$, for $\xi=0.1$

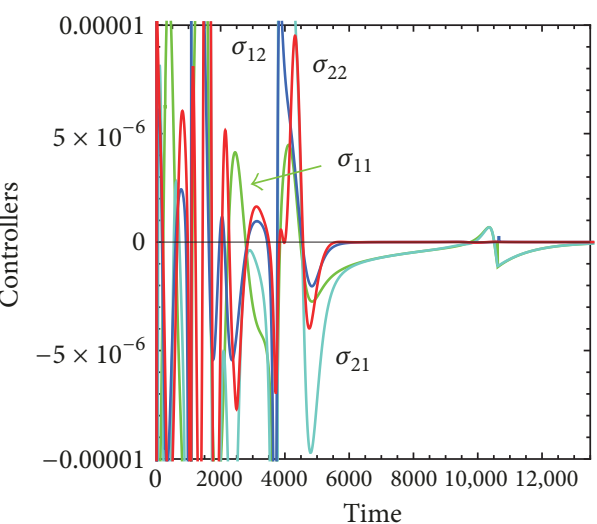

(d) Control signals, $\sigma_{i 1}$ and $\sigma_{i 2}$, for $\xi=0.2$

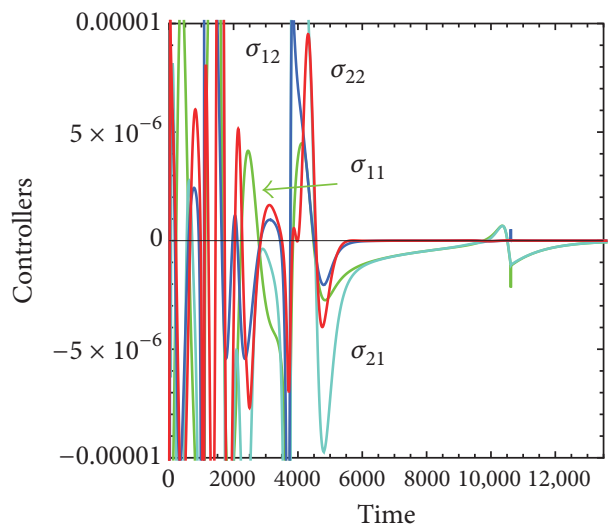

(f) Control signals, $\sigma_{i 1}$ and $\sigma_{i 2}$, for $\xi=0.5$

Figure 11: Trajectories of $\mathscr{A}_{1}$ and $\mathscr{A}_{2}$ and the evolution of control signals under various influences of noise.

desired relative distance $a_{k}$ and compares $B_{k}$ to the desired relative distance $b_{k}$, respectively. It is evident from the behavior shown in the figures that the formation gets slightly distorted when the C-ITS encounters an obstacle; however, the desired formation is reestablished before the leader $\mathscr{A}_{1}$ reaches its designated target, thus ensuring a locally rigid formation along the trajectory of the C-ITS.

\section{Conclusion}

This paper presents a set of nonlinear control laws using the LbCS to achieve a novel technique of extracting a locally rigid formation of a C-ITS made up of multiple car-like autonomous robots. A leader-following strategy is proposed to operate within the control scheme for the maintenance 
TABLE 2: Numerical values of initial and final states, constraints, and parameters for Scenario 3.

\begin{tabular}{|c|c|}
\hline & Initial configuration \\
\hline \multirow{4}{*}{ Rectangular positions } & $\left(x_{1}, y_{1}\right)=(7,20),\left(x_{2}, y_{2}\right)=(4,20),\left(x_{3}, y_{3}\right)=(7,23)$ \\
\hline & $\left(x_{4}, y_{4}\right)=(10,20)$, and $\left(x_{5}, y_{5}\right)=(7,17)$ \\
\hline & $\left(a_{2}, b_{2}\right)=(3,0),\left(a_{3}, b_{3}\right)=(0,-3)$ \\
\hline & $\left(a_{4}, b_{4}\right)=(-3,0)$, and $\left(a_{5}, b_{5}\right)=(0,3)$ \\
\hline \multirow[t]{2}{*}{ Velocity and angular position } & $v_{i}=0.5, \omega_{i}=0$, and $\theta_{i}=0$ for $i=1, \ldots, 5$ \\
\hline & Constraints and parameters \\
\hline Leader's target & $\left(p_{11}, p_{12}\right)=(57,37) ; r t=0.5$ \\
\hline \multirow[t]{2}{*}{ Final orientations } & $p_{i 3}=0$ for $i=1, \ldots, 5$ \\
\hline & Control and convergence parameters \\
\hline \multirow{3}{*}{ Obstacle avoidance } & $\alpha_{i l}=10$, for $i=1, \ldots, 5, l=1, \ldots, 3$ \\
\hline & $\kappa_{i p}=0.1$, for $i=1, \ldots, 5, p=1, \ldots, 2$ \\
\hline & $\gamma_{i k}=1.2 \times 10^{-4}$, for $i=1, \ldots, 5, k=1, \ldots, 3 i, k=1, \ldots, 3 n$ \\
\hline Collision avoidance & $\eta_{i j}=0.01$, for $i, j=1, \ldots, 5, j \neq i$ \\
\hline Dynamics constraints & $\beta_{i s}=0.001$, for $i=1, \ldots, 5, s=1,2$ \\
\hline Convergence & $\delta_{11}=20,500, \delta_{12}=10,500, \delta_{i 1}=60$, and $\delta_{i 2}=60$ for $i=1, \ldots, 5$ \\
\hline
\end{tabular}

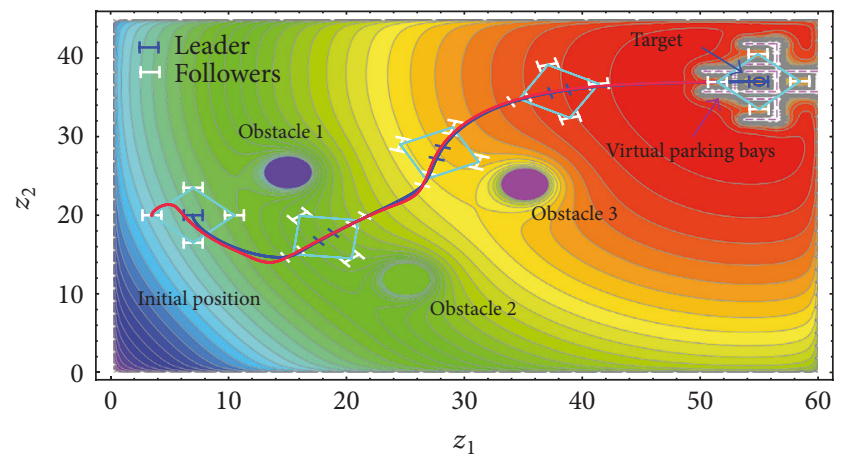

FIGURE 12: The evolution of the 5-robot C-ITS and the contour plot in the presence of obstacles in Scenario 3.

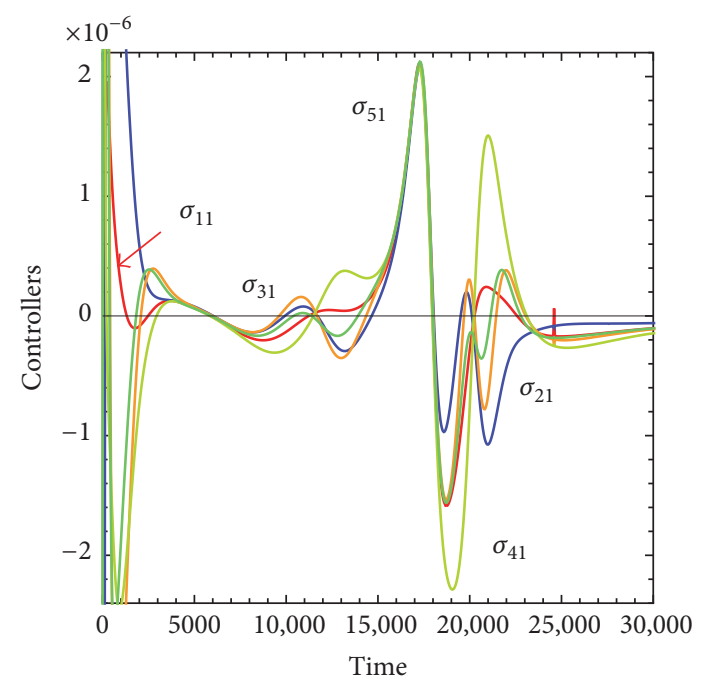

FIGURE 13: Translational accelerations, $\sigma_{1 i}$ for Scenario 3.

of the locally rigid formation for a C-ITS which navigates in a constrained environment. This leader-follower scheme

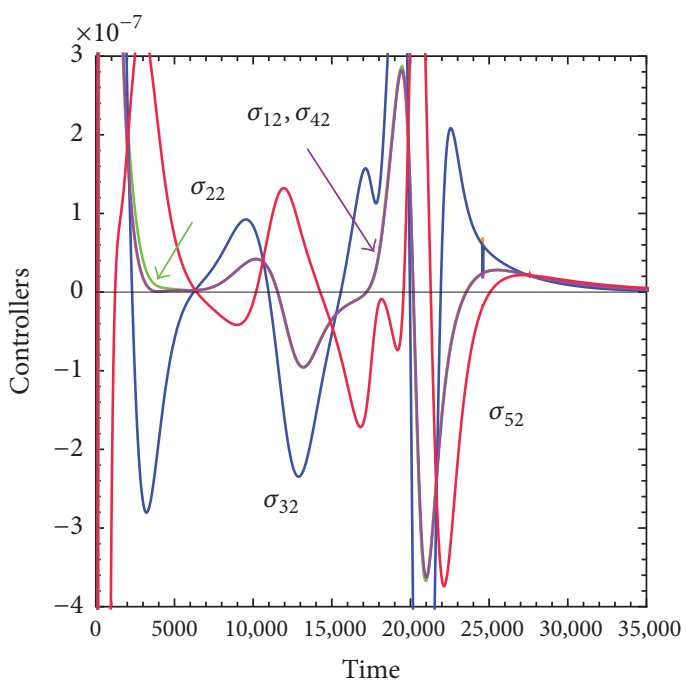

FIGURE 14: Rotational accelerations, $\sigma_{2 i}$ for Scenario 3.

uses a Cartesian coordinate system fixed on the leader's body based on the concept of an instantaneous corotating frame of reference to uniquely assign a position to each follower. The advantage of such an approach is the complete avoidance of singularities inherent to the polar coordinate representations.

The new time-invariant acceleration-based controllers produce feasible trajectories and ensured a nice convergence of the system to its equilibrium state while satisfying the necessary kinematic and dynamic constraints. The scheme enables the vehicles to follow a predetermined leader while maintaining a locally rigid formation. The robustness of the proposed scheme is established by considering the effect of noise on the formation, while stability of the system is guaranteed using the direct method of Lyapunov. The assumptions of no slippage and pure rolling of the car-like mobile robots are also considered to generate the nonholonomic constraints 


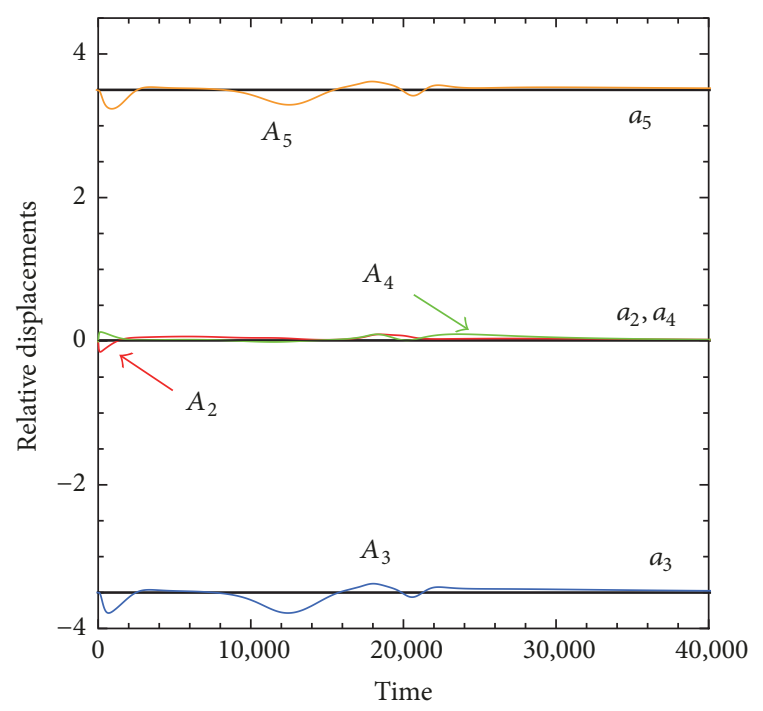

Figure 15: Relative distance error, $A_{k}$ to $a_{k}$ for Scenario 3.

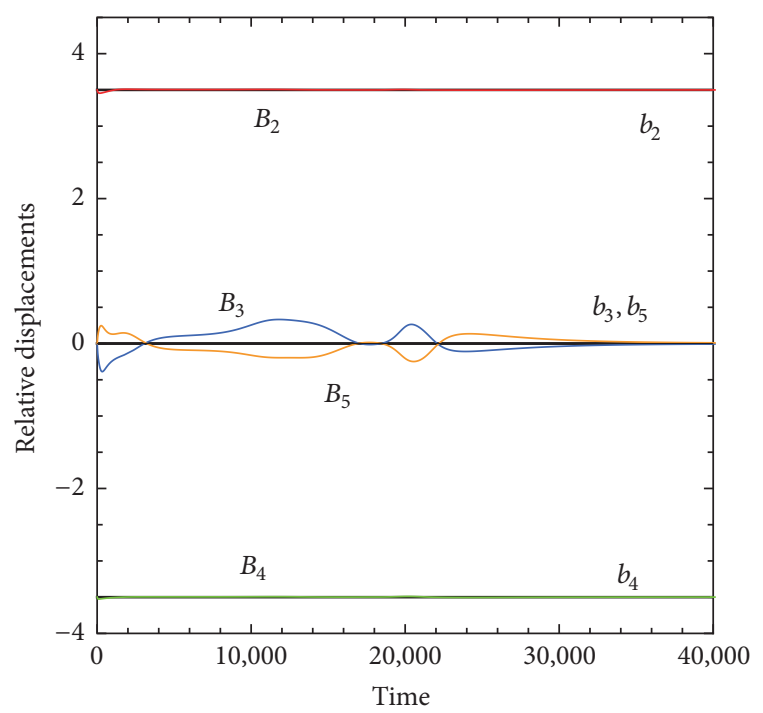

FIgURE 16: Relative distance error, $B_{k}$ to $b_{k}$ for Scenario 3.

on the given system. Further, the LbCS utilizes the minimum distance technique and parking bays to guarantee desired parking maneuvers and establish feasible prescribed posture of each vehicle in its designated parking bay.

This paper is a theoretical exposition into the applicability of an amalgamation of the new artificial potential field method, LbCS, and the leader-follower scheme. We restrict ourselves to showing the effectiveness of the control laws using computer-based simulations of interesting scenarios and numerical proofs. We shall be undertaking experiential design of the above system to see its effectiveness in the future. Future research will also address irregular shaped multimodal obstacles in partially known or completely known environments with the adaptive usage of sensing zones and relevant parameters. The optimization process of parameters within the LbCS still remains an open problem that warrants a careful thought.

\section{Appendix}

$$
\begin{aligned}
& f_{11}=\left[1+\sum_{l=1}^{q} \frac{\alpha_{1 l}}{\mathrm{FO}_{1 l}}+\sum_{s=1}^{2} \frac{\beta_{1 s}}{U_{1 s}}+\sum_{p=1}^{2} \frac{\kappa_{1 p}}{W_{1 p}}+\sum_{\substack{j=1 \\
j \neq i}}^{n} \frac{\eta_{1 j}}{\mathrm{MO}_{1 j}}\right. \\
& \left.+\sum_{k=1}^{3 n} \frac{\gamma_{1 k}}{L S_{1 k}}\right]\left(x_{1}-p_{11}\right)+\sum_{r=2}^{n}\left[1+\sum_{l=1}^{q} \frac{\alpha_{1 l}}{\mathrm{FO}_{1 l}}\right. \\
& \left.+\sum_{s=1}^{2} \frac{\beta_{1 s}}{U_{1 s}}\right]\left[-\left(A_{r}-a_{r}\right) \cos \theta_{1}+\left(B_{r}-b_{r}\right) \sin \theta_{1}\right] \\
& +\sum_{r=2}^{n}\left[\sum_{p=1}^{2} \frac{\kappa_{1 p}}{W_{1 p}}+\sum_{\substack{j=1 \\
j \neq i}}^{n} \frac{\eta_{1 j}}{\mathrm{MO}_{1 j}}+\sum_{k=1}^{3 n} \frac{\gamma_{1 k}}{L S_{1 k}}\right]\left[-\left(A_{r}\right.\right. \\
& \left.\left.-a_{r}\right) \cos \theta_{1}+\left(B_{r}-b_{r}\right) \sin \theta_{1}\right] \\
& -G_{1} \sum_{k=1}^{3} \frac{\gamma_{1 k}}{L S_{1 k}^{2}(\mathbf{x})}\left\{\left[1-\left(a_{k 2}-a_{k 1}\right) d_{k}\right]\left(x_{1}-L x_{1 k}\right)\right. \\
& \left.-\left(b_{k 2}-b_{k 1}\right) d_{k}\left(y_{1}-L y_{1 k}\right)\right\} \\
& -G_{1}\left[\sum_{l=1}^{q} \frac{\alpha_{1 l}}{\mathrm{FO}_{1 l}^{2}}\left(x_{1}-o_{l 1}\right)+2 \sum_{\substack{j=1 \\
j \neq i}}^{n} \frac{\eta_{1 j}}{\mathrm{MO}_{1 j}^{2}}\left(x_{1}\right.\right. \\
& \left.\left.-x_{j}\right)\right] \text {, } \\
& f_{12}=\left[1+\sum_{l=1}^{q} \frac{\alpha_{1 l}}{\mathrm{FO}_{1 l}}+\sum_{s=1}^{2} \frac{\beta_{1 s}}{U_{1 s}}+\sum_{p=1}^{2} \frac{\kappa_{1 p}}{W_{1 p}}+\sum_{\substack{j=1 \\
j \neq i}}^{n} \frac{\eta_{1 j}}{\mathrm{MO}_{1 j}}\right. \\
& \left.+\sum_{k=1}^{3 n} \frac{\gamma_{1 k}}{L S_{1 k}}\right]\left(y_{1}-p_{12}\right)+\sum_{h=2}^{n}\left[1+\sum_{l=1}^{q} \frac{\alpha_{1 l}}{\mathrm{FO}_{1 l}}\right. \\
& \left.+\sum_{s=1}^{2} \frac{\beta_{1 s}}{U_{1 s}}\right]\left[-\left(A_{h}-a_{h}\right) \sin \theta_{1}-\left(B_{h}-b_{h}\right) \cos \theta_{1}\right] \\
& +\sum_{h=2}^{n}\left[\sum_{p=1}^{2} \frac{\kappa_{1 p}}{W_{1 p}}+\sum_{\substack{j=1 \\
j \neq i}}^{n} \frac{\eta_{1 j}}{\mathrm{MO}_{1 j}}+\sum_{k=1}^{3 n} \frac{\gamma_{1 k}}{L S_{1 k}}\right]\left[-\left(A_{h}\right.\right. \\
& \left.\left.-a_{h}\right) \sin \theta_{1}-\left(B_{h}-b_{h}\right) \cos \theta_{1}\right]-G_{1}\left[\sum _ { l = 1 } ^ { q } \frac { \alpha _ { 1 l } } { \mathrm { FO } _ { 1 l } ^ { 2 } } \left(y_{1}\right.\right.
\end{aligned}
$$




$$
\begin{aligned}
\left.\left.-o_{l 2}\right)+2 \sum_{\substack{j=1 \\
j \neq i}}^{n} \frac{\eta_{1 j}}{\mathrm{MO}_{1 j}^{2}}\left(y_{1}-y_{j}\right)\right]-\frac{\kappa_{12}}{W_{12}^{2}}+\frac{\kappa_{14}}{W_{14}^{2}} \\
-G_{1} \sum_{k=1}^{3} \frac{\gamma_{1 k}}{L S_{1 k}^{2}(\mathbf{x})}\left\{\left[1-\left(b_{k 2}-b_{k 1}\right) r_{k}\right]\left(y_{1}-L y_{1 k}\right)\right. \\
\left.-\left(a_{k 2}-a_{k 1}\right) r_{k}\left(x_{1}-L x_{1 k}\right)\right\}, \\
f_{13}=\left[\sum_{l=1}^{q} \frac{\alpha_{1 l}}{\mathrm{FO}}+\sum_{1 l}^{2} \frac{\beta_{1 s}}{U_{1 s}}+\sum_{p=1}^{2} \frac{\kappa_{1 p}}{W_{1 p}}+\sum_{\substack{j=1 \\
j \neq i}}^{n} \frac{\eta_{1 j}}{\mathrm{MO}_{1 j}}\right. \\
+\sum_{k=1}^{3 n} \frac{\gamma_{1 k}}{L S_{1 k}} \rho_{1}\left(\theta_{1}-p_{13}\right)-\sum_{i=2}^{n}\left[\sum_{l=1}^{q} \frac{\alpha_{1 l}}{\mathrm{FO}_{1 l}}+\sum_{s=1}^{2} \frac{\beta_{1 s}}{U_{1 s}}\right. \\
\left.+\sum_{p=1}^{2} \frac{\kappa_{1 p}}{W_{1 p}}+\sum_{\substack{j=1 \\
j \neq i}}^{n} \frac{\eta_{1 j}}{\mathrm{MO}_{1 j}}+\sum_{k=1}^{3 n} \frac{\gamma_{1 k}}{L S_{1 k}}\right] \rho_{i}\left(\theta_{i}-\theta_{1}\right), \\
g_{11}=1+G_{1} \frac{\beta_{11}}{U_{11}^{2}}, \\
g_{12}=1+G_{1} \frac{\beta_{12}}{U_{12}^{2}},
\end{aligned}
$$

and, for $i=2, \ldots, n$,

$$
\begin{aligned}
& f_{i 1}=\left[1+\sum_{l=1}^{q} \frac{\alpha_{i l}}{\mathrm{FO}_{i l}}+\sum_{s=1}^{2} \frac{\beta_{i s}}{U_{i s}}+\sum_{p=1}^{2} \frac{\kappa_{i p}}{W_{i p}}+\sum_{\substack{j=1 \\
j \neq i}}^{n} \frac{\eta_{i j}}{\mathrm{MO}_{i j}}\right. \\
& \left.+\sum_{k=3 i-2}^{3 i} \frac{\gamma_{i k}}{L S_{i k}}\right]\left(A_{i}-a_{i}\right) \cos \theta_{1}-\left[1+\sum_{l=1}^{q} \frac{\alpha_{i l}}{\mathrm{FO}_{i l}}\right. \\
& \left.+\sum_{s=1}^{2} \frac{\beta_{i s}}{U_{i s}}+\sum_{p=1}^{2} \frac{\kappa_{i p}}{W_{i p}}+\sum_{\substack{j=1 \\
j \neq i}}^{n} \frac{\eta_{i j}}{\mathrm{MO}_{i j}}+\sum_{k=3 i-2}^{3 i} \frac{\gamma_{i k}}{L S_{i k}}\right]\left(B_{i}\right. \\
& \left.-b_{i}\right) \sin \theta_{1}-G_{i} \sum_{k=3 i-2}^{3 i} \frac{\gamma_{i k}}{L S_{i k}^{2}(\mathbf{x})}\{[1 \\
& \left.-\left(a_{k 2}-a_{k 1}\right) d_{k}\right]\left(x_{i}-L x_{i k}\right)-\left(b_{k 2}\right. \\
& \left.\left.-b_{k 1}\right) d_{k}\left(y_{i}-L y_{i k}\right)\right\} \\
& -G_{i}\left[\sum_{l=1}^{q} \frac{\alpha_{i l}}{\mathrm{FO}_{i l}^{2}}\left(x_{i}-o_{l 1}\right)+2 \sum_{\substack{j=1 \\
j \neq i}}^{n} \frac{\eta_{i j}}{\mathrm{MO}_{i j}^{2}}\left(x_{i}-x_{j}\right)\right],
\end{aligned}
$$

$$
\begin{aligned}
& f_{i 2}=\left[1+\sum_{l=1}^{q} \frac{\alpha_{i l}}{\mathrm{FO}_{i l}}+\sum_{s=1}^{2} \frac{\beta_{i s}}{U_{i s}}+\sum_{p=1}^{2} \frac{\kappa_{i p}}{W_{i p}}+\sum_{\substack{j=1 \\
j \neq i}}^{n} \frac{\eta_{i j}}{\mathrm{MO}_{i j}}\right. \\
& \left.+\sum_{k=1}^{3 n} \frac{\gamma_{i k}}{L S_{i k}}\right]\left(A_{i}-a_{i}\right) \sin \theta_{1}+\left[1+\sum_{l=1}^{q} \frac{\alpha_{i l}}{\mathrm{FO}_{i l}}\right. \\
& \left.+\sum_{s=1}^{2} \frac{\beta_{i s}}{U_{i s}}+\sum_{p=1}^{2} \frac{\kappa_{i p}}{W_{i p}}+\sum_{\substack{j=1 \\
j \neq i}}^{n} \frac{\eta_{i j}}{\mathrm{MO}_{i j}}+\sum_{k=1}^{3 n} \frac{\gamma_{i k}}{L S_{i k}}\right]\left(B_{i}\right. \\
& \left.-b_{i}\right) \cos \theta_{1}-G_{i} \sum_{k=3 i-2}^{3 i} \frac{\gamma_{i k}}{L S_{i k}^{2}(\mathbf{x})}\{[1 \\
& \left.-\left(b_{k 2}-b_{k 1}\right) r_{k}\right]\left(y_{i}-L y_{i k}\right)-\left(a_{k 2}-a_{k 1}\right) r_{k}\left(x_{i}\right. \\
& \left.\left.-L x_{i k}\right)\right\}-G_{i}\left[\sum_{l=1}^{q} \frac{\alpha_{i l}}{\mathrm{FO}_{i l}^{2}}\left(y_{i}-o_{l 1}\right)\right. \\
& \left.+2 \sum_{\substack{j=1 \\
j \neq i}}^{n} \frac{\eta_{i j}}{\mathrm{MO}_{i j}^{2}}\left(y_{i}-y_{j}\right)\right]-\frac{\kappa_{i 2}}{W_{i 2}^{2}}+\frac{\kappa_{i 4}}{W_{i 4}^{2}}, \\
& f_{i 3}=\left[\sum_{l=1}^{q} \frac{\alpha_{i l}}{\mathrm{FO}_{i l}}+\sum_{s=1}^{2} \frac{\beta_{i s}}{U_{i s}}+\sum_{p=1}^{2} \frac{\kappa_{i p}}{W_{i p}}+\sum_{\substack{j=1 \\
j \neq i}}^{n} \frac{\eta_{i j}}{\mathrm{MO}_{i j}}\right. \\
& \left.+\sum_{k=1}^{3 n} \frac{\gamma_{i k}}{L S_{i k}}\right] \rho_{i}\left(\theta_{i}-\theta_{1}\right) \\
& g_{i 1}=1+G_{i} \frac{\beta_{i 1}}{U_{i 1}^{2}} \\
& g_{i 2}=1+G_{i} \frac{\beta_{i 2}}{U_{i 2}^{2}} \text {. }
\end{aligned}
$$

\section{Conflicts of Interest}

The authors declare that they have no conflicts of interest.

\section{References}

[1] A. Festag, "Cooperative intelligent transport systems standards in Europe," IEEE Communications Magazine, vol. 52, no. 12, pp. 166-172, 2014.

[2] E. Ben Hamida, H. Noura, and W. Znaidi, "Security of cooperative intelligent transport systems: Standards, threats analysis 
and cryptographic countermeasures," Electronics, vol. 4, no. 3, pp. 380-423, 2015.

[3] T. Balch and M. Hybinette, "Social potentials for scalable multirobot formations," Proceedings-IEEE International Conference on Robotics and Automation, vol. 1, pp. 73-80, 2000.

[4] C. De La Cruz and R. Carelli, "Dynamic model based formation control and obstacle avoidance of multi-robot systems," Robotica, vol. 26, no. 3, pp. 345-356, 2008.

[5] F. Arrichiello, Coordination Control of Multiple Mobile Robots [Ph.D. thesis], Universita Degli Studi Di Cassino, Cassino, Italy, November 2006.

[6] T. H. A. Van Den Broek, N. Van De Wouw, and H. Nijmeijer, "Formation control of unicycle mobile robots: a virtual structure approach," in Proceedings of the 48th IEEE Conference on Decision and Control held jointly with 2009 28th Chinese Control Conference, CDC/CCC 2009, pp. 8328-8333, Shanghai, China, December 2009.

[7] H. Yamaguchi, "A distributed motion coordination strategy for multiple nonholonomic mobile robots in cooperative hunting operations," Robotics and Autonomous Systems, vol. 43, no. 4, pp. 257-282, 2003.

[8] Y. H. Esin and M. Unel, "Formation control if nonholonomic mobile robots using implicit polynomials and elliptic fourier descriptors," Turkish Journal of Electrical Engineering \& Computer Sciences, vol. 18, no. 5, pp. 765-80, 2010.

[9] R. Fierro, A. K. Das, V. Kumar, and J. P. Ostrowski, "Hybrid control of formations of robots," in Proceedings of the IEEE Conference Robotics and Automation, vol. 1, pp. 374-379, Seoul, Korea, May 2001.

[10] W. L. Seng, J. C. Barca, and Y. A. Şekercioğlu, "Distributed formation control of networked mobile robots in environments with obstacles," Robotica, vol. 12, pp. 1-13, 2014.

[11] F. Fahimi, "Full formation control for autonomous helicopter groups," Robotica, vol. 26, no. 2, pp. 143-156, 2008.

[12] R. Olfati-Saber, "Flocking for multi-agent dynamic systems: algorithms and theory," Institute of Electrical and Electronics Engineers Transactions on Automatic Control, vol. 51, no. 3, pp. 401-420, 2006.

[13] R. Falconi, L. Sabattini, C. Secchi, C. Fantuzzi, and C. Melchiorri, "Edge-weighted consensus-based formation control strategy with collision avoidance," Robotica, vol. 33, no. 2, pp. 332-347, 2015.

[14] B. Sharma, New Directions in the Applications of the Lyapunovbased Control Scheme to the Findpath Problem [Ph.D. thesis], University of the South Pacific, Suva, Fiji Islands, July 2008.

[15] E. Yang, D. Gu, and H. Hu, "Nonsingular formation control of cooperative mobile robots via feedback linearization," in Proceedings of the IEEE IRS/RSJ International Conference on Intelligent Robots and Systems (IROS '05), pp. 3652-3657, August 2005.

[16] W. Kang, N. Xi, J. Tan, and Y. Wang, "Formation control of multiple autonomous robots:theory and experimentation," Intelligent Automation \& Soft Computing, vol. 10, no. 2, pp. 117, 2004.

[17] A. Bazoula, M. S. Djouadi, and H. Maaref, "Formation control of multi-robots via fuzzy logic technique," International Journal of Computers, Communications \& Control, vol. 3, pp. 179-184, 2008.

[18] N. H. M. Li and H. H. T. Liu, "Formation UAV flight control using virtual structure and motion synchronization," in Proceedings of the 2008 American Control Conference (ACC'08), pp. 1782-1787, Washington, Wash, USA, June 2008.
[19] W. Ren and R. W. Beard, "Virtual structure based spacecraft formation control with formation feedback," in Proceedings of the AIAA Guidance, Navigation, and Control Conference and Exhibit, California, Calif, USA, August 2002.

[20] M. Egerstedt and X. Hu, "Formation constrained multi-agent control," in Proceedings of the 2001 IEEE International Conference on Robotics and Automation (ICRA '01), pp. 3961-3966, Seoul, Republic of Korea, May 2001.

[21] N. Sorensen and W. Ren, "A unified formation control scheme with a single or multiple leaders," in Proceedings of the 2007 American Control Conference (ACC '07), pp. 5412-5418, New York, NY, USA, July 2007.

[22] L. Consolini, F. Morbidi, D. Prattichizzo, and M. Tosques, "Leader-follower formation control of nonholonomic mobile robots with input constraints," Automatica, vol. 44, no. 5, pp. 1343-1349, 2008.

[23] W. J. Dong, Y. Guo, and J. A. Farrell, "Formation control of nonholonomic mobile robots," in Proceedings of the 2006 American Control Conference, pp. 5902-5607, Minneapolis, MN, USA, June 2006.

[24] K. Raghuwaiya and S. Singh, "Formation types of multiple steerable 1-trailer mobile robots via split/rejoin maneuvers," New Zealand Journal of Mathematics, vol. 43, pp. 7-21, 2013.

[25] B. Sharma, J. Vanualailai, and A. Prasad, "Formation control of a swarm of mobile manipulators," Rocky Mountain Journal of Mathematics, vol. 41, no. 3, pp. 909-940, 2011.

[26] B. Sharma, J. Vanualailai, and A. Prasad, "A $d \phi$ strategy: facilitating dual-formation control of a virtually connected team," Journal of Advanced Transportation, vol. 2017, Article ID 9213805, 17 pages, 2017.

[27] K. Raghuwaiya, B. Sharma, and J. Vanualailai, Advanced Computer and Communication Engineering Technology: Lecture Notes in Electrical Engineering, Cooperative Control of Multi Robot Systems with a low-degree formation, Springer, 2015.

[28] R. W. Brockett, Differential Geometric Control Theory, Asymptotic Stability and Feedback Stabilisation, Springer, 1983.

[29] A. Prasad, B. Sharma, and J. Vanualailai, "A new stabilizing solution for motion planning and control of multiple robots," Robotica, vol. 34, no. 5, pp. 1071-1089, 2016. 


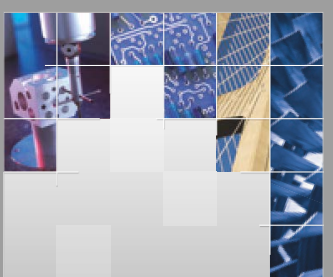

\section{Enfincering}
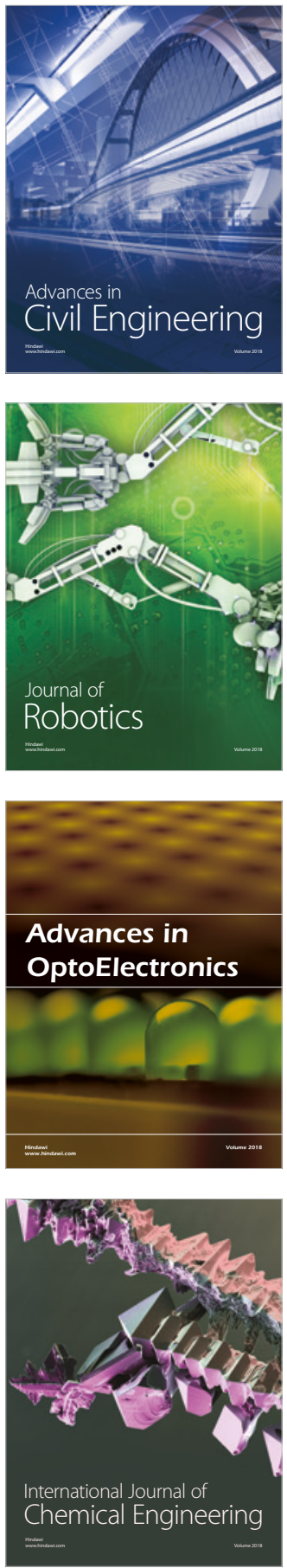

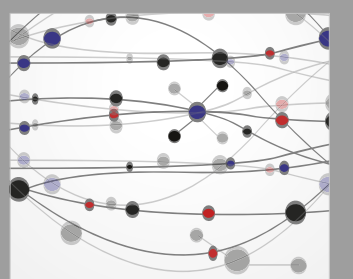

\section{Rotating \\ Machinery}

The Scientific World Journal

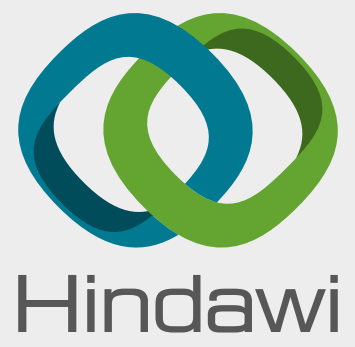

Submit your manuscripts at

www.hindawi.com
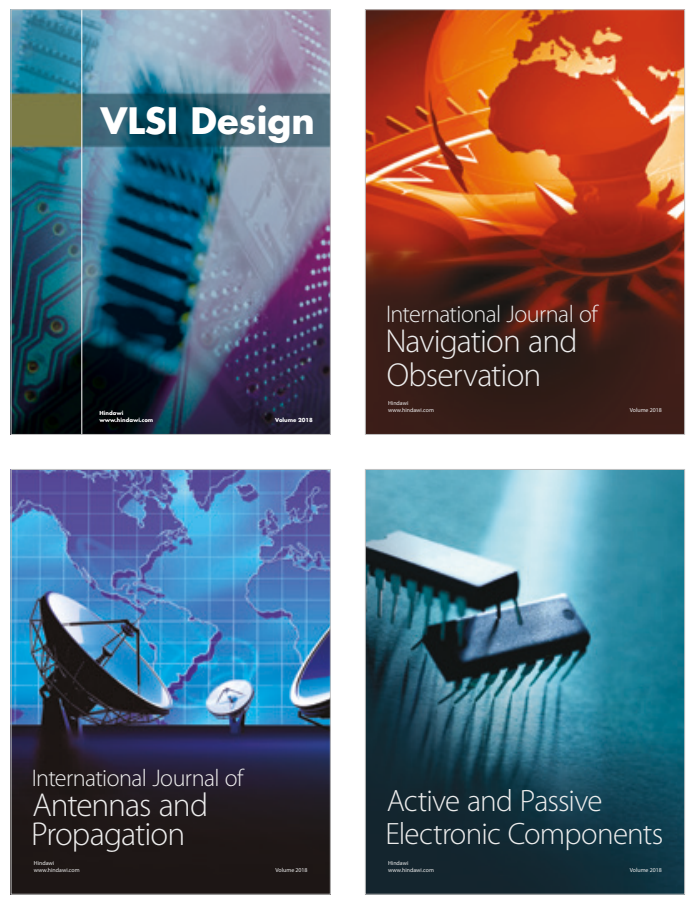
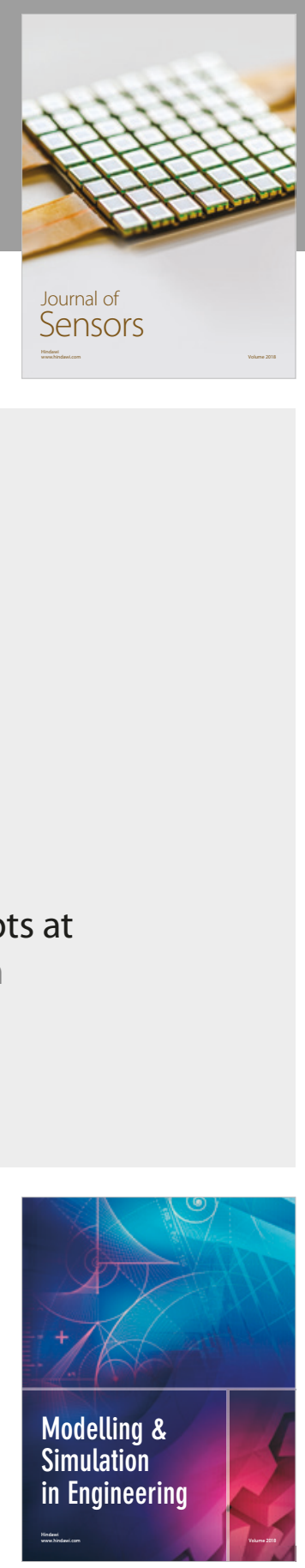

\section{Advances \\ Multimedia}
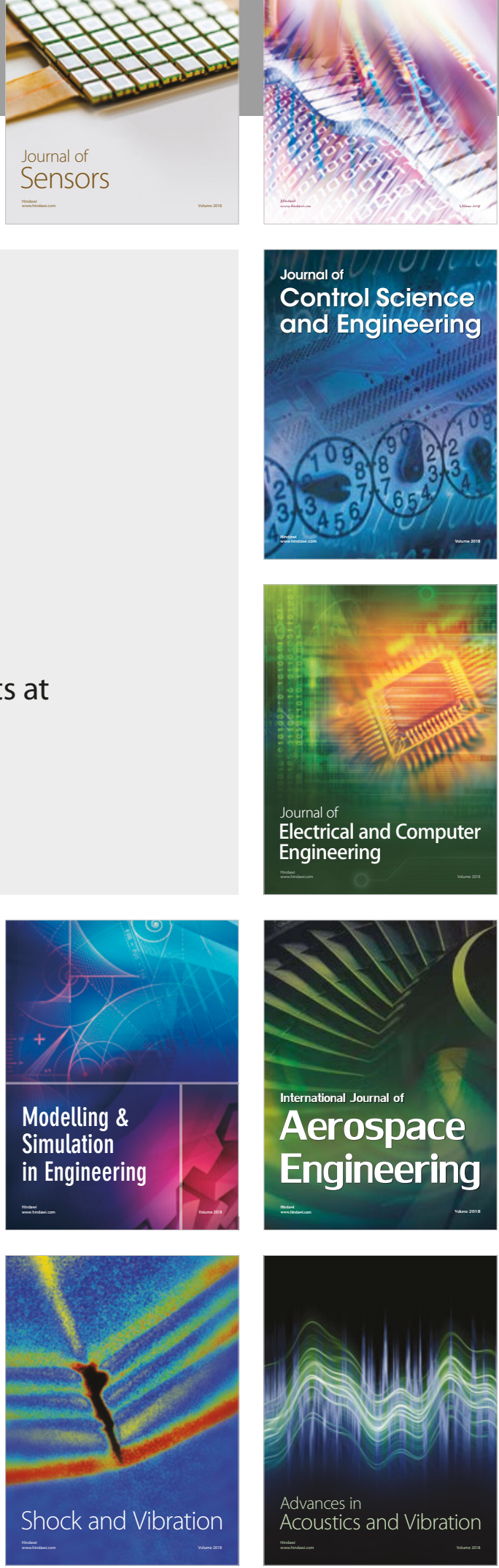